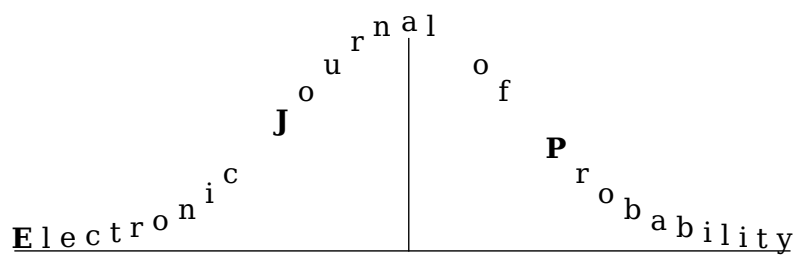

Electron. J. Probab. 25 (2020), no. 51, 1-26.

ISSN: 1083-6489 https://doi.org/10.1214/20-EJP451

\title{
Compactness and continuity properties for a Lévy process at a two-sided exit time ${ }^{* \dagger}$
}

\author{
Ross A. Maller $\quad$ David M. Mason ${ }^{\S}$
}

\begin{abstract}
We consider a Lévy process $X=(X(t))_{t \geq 0}$ in a generalised Feller class at 0 , and study the exit position, $|X(T(r))|$, as $X$ leaves, and the position, $|X(T(r)-)|$, just prior to its leaving, at time $T(r)$, a two-sided region with boundaries at $\pm r, r>0$. Conditions are known for $X$ to be in the Feller class $F C_{0}$ at zero, by which we mean that each sequence $t_{k} \downarrow 0$ contains a subsequence through which $X\left(t_{k}\right)$, after norming by a nonstochastic function, converges to an a.s. finite nondegenerate random variable. We use these conditions on $X$ to characterise similar properties for the normed positions $|X(T(r))| / r$ and $|X(T(r)-)| / r$, and also for the normed jump $|\Delta X(T(r)) / r|=|X(T(r))-X(T(r)-)| / r$ ("the jump causing ruin"), as convergence takes place through sequences $r_{k} \downarrow 0$. We go on to give conditions for the continuity of distributions of the limiting random variables obtained in this way.
\end{abstract}

Keywords: Lévy process; passage time distributions; two-sided exit problem; domain of partial attraction; generalised Feller class; stochastic compactness.

AMS MSC 2010: 62E17; 62B15; $62 \mathrm{G} 05$.

Submitted to EJP on July 26, 2019, final version accepted on April 7, 2020.

\section{Introduction and background}

Feller in [9] generalised the concept of domains of attraction for random walks by considering certain kinds of convergence of the normed and centered sums of i.i.d random variables through subsequences, thereby introducing the class of stochastically compact random walks. In a similar vein, [15] considered Lévy processes $X=(X(t))_{t \geq 0}$, $X(0)=0$, on $\mathbb{R}$, which have this kind of behavior, after norming and centering, as $t \downarrow 0$. They called this class $F C$ (at 0 ), and also introduced a subclass, denoted $F C_{0}$, in which centering is not required for such an $X$. See Subsection 1.4 for further information on these classes.

\footnotetext{
*David Mason is pleased to acknowledge support from the Research School of Finance, Actuarial Studies and Statistics at the Australian National University, on a number of visits to Canberra.

${ }^{\dagger}$ This article was first posted online with the misprints in the second to last paragraph in section 1.1. Misprints have been corrected on 19 May 2020.

${ }^{\ddagger}$ Research School of Finance, Actuarial Studies and Statistics, Australian National University, Canberra, ACT, 0200, Australia. E-mail: Ross.Maller@anu. edu .au

${ }^{\S}$ Department of Applied Economics and Statistics, University of Delaware, 206 Townsend Hall, Newark, DE 19717, USA. E-mail: davidm@udel .edu
} 


\subsection{The two-sided exit problem}

We continue the study of the small time behavior of a nonzero Lévy process by investigating the position of the process $X$ at exit and just before exit from a two-sided region, defined in terms of horizontal boundaries. Thus we consider the two-sided exit time $T(r)$ defined as

$$
T(r)=\inf \{t>0:|X(t)|>r\}, r>0 .
$$

Since $X$ is nonzero, $\lim \sup _{t \rightarrow \infty}|X(t)|=\infty$ almost surely (a.s.), and so $T(r)<\infty$ a.s. for each $r>0$. By right continuity of $(X(t))_{t \geq 0}$, which we assume as usual, we have $0=X(0)=X(0+)=\lim _{t \downarrow 0} X(t)$, so $|X(t)|<r$ a.s. for $t$ sufficiently small, and so $T(r)>0$ a.s. for each $r>0$. Thus we can define the position of the process at exit, $X(T(r))$, and the position just before exit, $X(T(r)-)$, and then the size of the jump at exit ("the jump causing ruin"),

$$
\Delta X(T(r)):=X(T(r))-X(T(r)-), r>0 .
$$

As $r \downarrow 0, T(r) \downarrow 0$ a.s. and $X(T(r)) \rightarrow 0$ a.s., and the size of $|X(T(r))|$ by comparison with the boundary value $r$ is of interest. Our aim is to investigate the stochastic compactness of $|X(T(r))| / r,|X(T(r)-)| / r$, and $|\Delta X(T(r))| / r$ as $r \downarrow 0$. By this we mean the convergence in distribution of these quantities to finite nondegenerate limits through subsequences of $r$ tending to 0 . The properties we consider are closely connected to stochastic compactness properties of $X(t)$ itself, as might be expected, but there are some notable distinctions that need to be teased out. Furthermore, from these, we study properties of the subsequential limit random variables (rvs) of $|X(T(r))| / r,|X(T(r)-)| / r$ and $|\Delta X(T(r))| / r$, for small $r$, giving conditions, in particular, for the continuity of their subsequential limit distributions, and, in the case of $|X(T(r))| / r$, a condition for Lipschitz and absolute continuity of the limit distributions.

We remark in passing that the analogs of our results are true for the stochastic compactness of $|X(T(r))| / r,|X(T(r)-)| / r$, and $|\Delta X(T(r))| / r$ as $r \uparrow \infty$. The proofs follow similarly as for the case $r \downarrow 0$ with small changes. The requisite Feller class of $X$ at $\infty$ results are detailed in [16].

\subsection{Lévy process setup, notation and preliminary results}

Consider a Lévy process $(X(t))_{t \geq 0}$, having Lévy triplet $\left(\gamma, \sigma^{2}, \Pi\right)$, thus, having infinitely divisible (inf. div.) characteristic function (c.f.)

$$
E e^{\mathrm{i} \theta X(t)}=e^{t \Psi(\theta)}, \quad t>0, \theta \in \mathbb{R},
$$

where

$$
\Psi(\theta)=\mathrm{i} \gamma \theta-\frac{1}{2} \sigma^{2} \theta^{2}+\int_{\mathbb{R} \backslash\{0\}}\left(e^{\mathrm{i} \theta x}-1-\mathrm{i} \theta x \mathbf{1}_{\{|x| \leq 1\}}\right) \Pi(\mathrm{d} x),
$$

with $\gamma \in \mathbb{R}, \sigma^{2} \geq 0$, and $\Pi$ a measure on $\mathbb{R}$ with $\int_{\mathbb{R} \backslash\{0\}}\left(x^{2} \wedge 1\right) \Pi(\mathrm{d} x)$ finite. Define the positive, negative and two-sided Lévy tail functions

$$
\bar{\Pi}^{+}(x)=\Pi\{(x, \infty)\}, \bar{\Pi}^{-}(x)=\Pi\{(-\infty,-x)\} \text {, and } \bar{\Pi}(x)=\bar{\Pi}^{+}(x)+\bar{\Pi}^{-}(x), x>0 .
$$

Throughout, we assume $\sigma^{2}>0$ or $\Pi(\mathbb{R} \backslash\{0\})>0$, so $X$ is not the process degenerate at 0 . In fact, for most of our results we will assume $\Pi(\mathbb{R} \backslash\{0\})=\bar{\Pi}(0+)=\infty$, so $X$ is not a compound Poisson process.

We will need a truncated mean function defined by

$$
\nu(x)=\left\{\begin{array}{l}
\gamma-\int_{x<|y| \leq 1} y \Pi(\mathrm{d} y), 0<x \leq 1, \\
\gamma+\int_{1<|y| \leq x} y \Pi(\mathrm{d} y), x>1,
\end{array}\right.
$$


and truncated second moment functions defined for $x>0$ by

$$
V(x)=\sigma^{2}+\int_{0<|y| \leq x} y^{2} \Pi(\mathrm{d} y) \quad \text { and } \quad U(x)=\sigma^{2}+2 \int_{0}^{x} y \bar{\Pi}(y) \mathrm{d} y=V(x)+x^{2} \bar{\Pi}(x) .
$$

Also introduce the function

$$
h(x)=\frac{x|\nu(x)|+U(x)}{x^{2}}, x>0 .
$$

Properties of $h(x)$ can be deduced from results given in [19]. In particular, assuming as we do that $\sigma^{2}>0$ or $\Pi(\cdot)$ is not identically 0 , we have, for $x>0$ and $\lambda>1$,

$$
\lambda^{-2} h(x) / 2 \leq h(\lambda x) \leq 3 h(x) .
$$

(See also Lemma 2 of [8].)

\subsection{Convergence criteria}

Let $(X(t))_{t \geq 0}$ be a Lévy process having Lévy triplet $\left(\gamma, \sigma^{2}, \Pi\right)$, thus, having characteristic exponent (1.1). It's easy to check then that, for $t_{k}>0, a_{k} \in \mathbb{R}$ and $b_{k}>0$, $\left(X\left(t_{k}\right)-a_{k}\right) / b_{k}$ is an infinitely divisible random variable with triplet $\left(\gamma_{k}, \sigma_{k}^{2}, \Pi_{k}\right)$, where

$$
\sigma_{k}^{2}=\frac{\sigma^{2} t_{k}}{b_{k}^{2}}, \gamma_{k}=\frac{\gamma t_{k}-a_{k}}{b_{k}}+\int_{\mathbb{R} \backslash\{0\}} x\left(\mathbf{1}_{\{|x| \leq 1\}}-\mathbf{1}_{\left\{\left|b_{k} x\right| \leq 1\right\}}\right) \Pi_{k}(\mathrm{~d} x),
$$

and $\Pi_{k}(\mathrm{~d} x)=t_{k} \Pi\left(b_{k} \mathrm{~d} x\right)$. Theorem 15.14, p.295, of [13] says that

$$
\frac{X\left(t_{k}\right)-a_{k}}{b_{k}} \stackrel{\mathrm{D}}{\longrightarrow} Y, \text { as } k \rightarrow \infty,
$$

where $Y$ is an inf. div. rv with triplet $\left(\beta, \tau^{2}, \Lambda\right)$ such that $\beta \in \mathbb{R}, \tau^{2} \geq 0$, and $\Lambda$ is a Lévy measure on $\mathbb{R}$, if and only if for all $h>0$ such that $\pm h$ are points of continuity of $\Lambda(\cdot)$ :

$$
\begin{gathered}
\lim _{k \rightarrow \infty} t_{k} \bar{\Pi}^{ \pm}\left(h b_{k}\right)=\bar{\Lambda}^{ \pm}(h), \\
\sigma_{k}^{2}+\int_{|x|<h} x^{2} \Pi_{k}(\mathrm{~d} x)=\frac{t_{k}}{b_{k}^{2}} V\left(h b_{k}\right) \rightarrow \tau^{2}+\int_{0<|x| \leq h} x^{2} \Lambda(\mathrm{d} x),
\end{gathered}
$$

and

$$
\gamma_{k}-\int_{h<|x| \leq 1} x t_{k} \Pi\left(b_{k} \mathrm{~d} x\right)=\frac{t_{k} \nu\left(h b_{k}\right)-a_{k}}{b_{k}} \rightarrow \beta-\int_{h<|x| \leq 1} x \Lambda(\mathrm{d} x) .
$$

A consequence of (1.8) is that

$$
\lim _{k \rightarrow \infty} t_{k} \bar{\Pi}\left(h b_{k}\right)=\bar{\Lambda}(h):=\bar{\Lambda}^{+}(h)+\bar{\Lambda}^{-}(h)
$$

for all $h>0$ such that $h$ is a point of continuity of $\bar{\Lambda}$. We can replace the function $V$ in (1.9) by $U$ with obvious modifications, using (1.4). For a $d$-dimensional statement of these criteria refer to [18 $]^{1}$.

As special cases, equivalent to

$$
X\left(t_{k}\right) / b_{k} \stackrel{P}{\rightarrow} \pm 1
$$

for given nonstochastic sequences $t_{k} \downarrow 0$ and $b_{k}>0$, is, for all $h>0$,

$$
\lim _{k \rightarrow \infty} t_{k} \bar{\Pi}\left(h b_{k}\right)=0, \lim _{k \rightarrow \infty} \frac{t_{k} \nu\left(h b_{t}\right)}{b_{k}}= \pm 1, \lim _{k \rightarrow \infty} \frac{t_{k} V\left(h b_{k}\right)}{b_{k}^{2}}=0,
$$

\footnotetext{
${ }^{1}$ In their equation (7.11) and Lemma 7.5, $\mathbf{b}_{t}$ should be $\mathbf{b}_{t} / t$ and, in equation (7.15), $\mathbf{b}_{t_{k}}$ should be $\mathbf{b}_{t_{k}} / t_{k}$.
} 
and equivalent to $\left(X\left(t_{k}\right)-a_{k}\right) / b_{k} \stackrel{\mathrm{D}}{\longrightarrow} N(0,1)$ for given nonstochastic sequences $t_{k} \downarrow 0$, $a_{k} \in \mathbb{R}$ and $b_{k}>0$, is, for all $h>0$,

$$
\lim _{k \rightarrow \infty} t_{k} \bar{\Pi}\left(h b_{k}\right)=0, \lim _{k \rightarrow \infty} \frac{t_{k} \nu\left(h b_{k}\right)-a_{k}}{b_{k}}=0, \lim _{k \rightarrow \infty} \frac{t_{k} V\left(h b_{k}\right)}{b_{k}^{2}}=1 .
$$

Here and throughout, $N(a, b)$ denotes a normal random variable with mean $a \in \mathbb{R}$ and variance $b>0$.

Obvious modifications of (1.8)-(1.10) characterize convergence through a continuous limit as $t \downarrow 0$.

\subsection{Feller class for $X$ at 0}

As in [15], we say that $X$ is in the Feller class at zero, written $X(t) \in F C$ at 0 , if there exist non-stochastic functions $a(t) \in R$ and $b(t)>0$ such that each sequence $t_{k} \downarrow 0$ contains a subsequence $t_{k^{\prime}} \downarrow 0$ for which

$$
\frac{X\left(t_{k^{\prime}}\right)-a\left(t_{k^{\prime}}\right)}{b\left(t_{k^{\prime}}\right)} \stackrel{\mathrm{D}}{\longrightarrow} Y^{\prime}, \text { as } t_{k^{\prime}} \downarrow 0
$$

where $Y^{\prime}$ is an a.s. finite random variable which is not degenerate at any constant, and may depend on the choice of subsequence $t_{k^{\prime}}$.

When $a(t)$ may be taken as 0 , we say that $X$ is in the centered Feller class at zero, written $X(t) \in F C_{0}$ at 0 . Specifically, $X \in F C_{0}$ at 0 if there exists a non-stochastic function $b(t)>0$ such that each sequence $t_{k} \downarrow 0$ contains a subsequence $t_{k^{\prime}} \downarrow 0$ for which

$$
\frac{X\left(t_{k^{\prime}}\right)}{b\left(t_{k^{\prime}}\right)} \stackrel{\mathrm{D}}{\longrightarrow} Y^{\prime}, \text { as } t_{k^{\prime}} \downarrow 0
$$

where $Y^{\prime}$ is an a.s. finite random variable which is not degenerate at any constant, and may depend on the choice of subsequence $t_{k^{\prime}}$. We describe $Y^{\prime}$ in (1.12) or (1.13) as a "subsequential limit rv" of the rescaled $X$, as $t \downarrow 0$.

[15] give the following analytic equivalences for $F C$ and $F C_{0}$ at 0.

Theorem 1.1. Assume $\sigma^{2}>0$ or $\bar{\Pi}(0+)=\infty$. Then

(i) $X(t) \in F C$ at 0 if and only if

$$
\limsup _{x \downarrow 0} \frac{x^{2} \bar{\Pi}(x)}{V(x)}<\infty ;
$$

(ii) $X(t) \in F C_{0}$ at 0 if and only if

$$
\limsup _{x \downarrow 0} \frac{x^{2} \bar{\Pi}(x)+x|\nu(x)|}{V(x)}<\infty .
$$

When (1.12) or (1.13) holds, a functional version also holds; in particular, when $X(t) \in$ $F C_{0}$ we can strengthen the convergence in (1.13) to

$$
\left(\frac{X\left(s t_{k^{\prime}}\right)}{b\left(t_{k^{\prime}}\right)}\right)_{0<s \leq T} \stackrel{\mathrm{D}}{\longrightarrow}\left(Y^{\prime}(s)\right)_{0<s \leq T} \text {, as } t_{k^{\prime}} \downarrow 0,
$$

in $D[0, T]$ for any fixed $T>0$, where $\left(Y^{\prime}(s)\right)_{0<s \leq T}$ is a Lévy process with $Y^{\prime}(1) \stackrel{\mathrm{D}}{=} Y^{\prime}$.

Moreover, when (1.12) or (1.13) holds, we can choose a norming function $b(t)>0$ satisfying, for each small enough $t>0$,

$$
\frac{U(b(t))}{b^{2}(t)}=\frac{1}{t}
$$


Although not stated in [15], it can be proved using similar methods that if (1.12) holds with functions $a(t)$ and $b(t)$ replaced by functions $c(t)$ and $d(t)$, then $d(t) \asymp b(t)$ as $t \downarrow 0$ in the sense that $0<\liminf _{t \downarrow 0} d(t) / b(t) \leq \lim \sup _{t \downarrow 0} d(t) / b(t)<\infty$; and similarly for the version when $a(t)$ is taken as 0 . We remark that the function $x \mapsto x^{2} / U(x)$ used in (1.17) is strictly increasing and takes value 0 at $x=0$ and value $\infty$ at $x=\infty$, under our assumptions, hence has a finite, uniquely defined inverse on $(0, \infty)$. The function $b(t)$ is this inverse function, evaluated at $t, t>0$.

Remark 1.2. Various properties of the subsequential limit rvs in (1.12) and (1.13) have been derived. For example, any such $Y^{\prime}$ is infinitely divisible with an absolutely continuous distribution ([5]), and the same is true for each $Y^{\prime}(s)$ for every $s>0$.

We emphasize, however, relevant to our discussion, that the Lévy measure of $Y^{\prime}$ is not in general continuous; a counterexample using a symmetric St. Petersburg process is detailed in Section 5.

\subsection{Partial attraction to normality}

We say that $X$ is in the domain of partial attraction of the normal distribution at 0 , written as $X(t) \in D_{P}(N)$ at 0 , if there exist sequences $t_{k} \downarrow 0, a_{k} \in \mathbb{R}$ and $b_{k}>0$ such that

$$
\frac{X\left(t_{k}\right)-a_{k}}{b_{k}} \stackrel{\mathrm{D}}{\longrightarrow} N(a, b), \text { as } k \rightarrow \infty,
$$

for finite constants $a \in \mathbb{R}$ and $b>0$. The condition $X(t) \in D_{P}(N)$ will play an important role in the many of the statements of our main results. In Subsection 1.3 we gave criteria for (1.18) to occur, for designated sequences $a_{k}$ and $b_{k}>0$ (see (1.11)). We can also ask for an existence result; for a given Lévy process with triplet $\left(\gamma, \sigma^{2}, \Pi\right)$, when are there sequences producing the behavior in (1.18)? Such criteria are given for $D_{P}(N)$ in [14], and also for the class $D_{P 0}(N)$ (see Theorems 1 and 2 of [14]), where we say that $X$ is in $D_{P 0}(N)$ at 0 , the centered domain of partial attraction of the normal distribution, if (1.18) holds for some $b_{k}>0, a \in \mathbb{R}, b>0$, but with $a_{k}=0$. We list these analytic criteria here as:

Theorem 1.3. Assume $\sigma^{2}>0$ or $\bar{\Pi}(0+)=\infty$.

(i) $X(t) \in D_{P}(N)$ at 0 if and only if

$$
\limsup _{x \downarrow 0} \frac{V(x)}{x^{2} \bar{\Pi}(x)}=\infty .
$$

(ii) $X(t) \in D_{P 0}(N)$ at 0 if and only if

$$
\limsup _{x \downarrow 0} \frac{V(x)}{x^{2} \bar{\Pi}(x)+x|\nu(x)|}=\infty .
$$

By (1.4) we can replace $V$ by $U$ in (1.19) and (1.20), to get the forms in which they are stated in [14]. See also Theorem 6.1 of [17] where a $d$-dimensional version of (1.19) is given.

\subsection{Distributions and inequalities for positions and jump size}

Here we state some identities and inequalities concerning the distributions of $X(T(r))$, $X(T(r)-)$, and $\Delta X(T(r))$. Important to our analyses are versions for Lévy processes of inequalities of [19]. They state that there exist constants $c_{1}>0$ and $C_{1}>0$, not depending on $t$ or $r$, such that, for all $t>0$ and $r>0$

$$
P\left(\sup _{0 \leq s \leq t}|X(s)| \leq r\right) \leq \frac{c_{1}}{t h(r)},
$$


where $h(\cdot)$ is the function in (1.5), and

$$
P\left(\sup _{0 \leq s \leq t}|X(s)| \geq r\right) \leq C_{1} \operatorname{th}(r)
$$

and there exist constants $c_{2}>0$ and $C_{2}>0$, not depending on $r$, such that, for all $r>0$,

$$
\frac{c_{2}}{h(r)} \leq E T(r) \leq \frac{C_{2}}{h(r)}
$$

(See also Lemma 1 of [8].) We will also need the function

$$
U_{r}(d y):=\int_{0}^{\infty} P\left(\sup _{0 \leq u<t}|X(u)| \leq r, X(t-) \in \mathrm{d} y\right) \mathrm{d} t,-r \leq y \leq r,
$$

and the associated measure

$$
\begin{gathered}
U_{r}((a, b)):=\int_{a<y<b} U_{r}(d y) \\
=\int_{0}^{\infty} P\left(\sup _{0 \leq u<t}|X(u)| \leq r, a<X(t-)<b\right) \mathrm{d} t,-r \leq a<b \leq r .
\end{gathered}
$$

Notice from (1.23) that for $r>0$

$$
\begin{gathered}
U_{r}([-r, r])=\int_{0}^{\infty} P\left(\sup _{0 \leq u<t}|X(u)| \leq r,|X(t)| \leq r\right) \mathrm{d} t \\
=\int_{0}^{\infty} P\left(\sup _{0 \leq u \leq t}|X(u)| \leq r\right) \mathrm{d} t=\int_{0}^{\infty} P(T(r)>t) \mathrm{d} t=E(T(r)) .
\end{gathered}
$$

In the next lemma, Part (iii) is from [7], and we add in results for the position at exit, $X(T(r))$, and the position just before exit, $X(T(r)-)$. Recall the definitions of $\bar{\Pi}^{ \pm}(x)$ in (1.2), and note the obvious properties $|X(T(r))| \geq 1$ and $|X(T(r)-)| \leq 1$.

Lemma 1.4. Suppose $\bar{\Pi}(0+)>0$ and $r>0$. Then

(i) For $s>1$

$$
P(|X(T(r))|>r s)=\int_{|y| \leq r}\left(\bar{\Pi}^{+}(r s-y)+\bar{\Pi}^{-}(r s+y)\right) U_{r}(\mathrm{~d} y) .
$$

(ii) For $0<s<1$

$$
P(|X(T(r)-)| \leq r s)=\int_{|y| \leq r s}\left(\bar{\Pi}^{+}(r-y)+\bar{\Pi}^{-}(r+y)\right) U_{r}(\mathrm{~d} y) .
$$

(iii) For $0<x \leq 2 r$

$$
\begin{aligned}
P(|\Delta X(T(r))|>x) & =\left(\int_{-r \leq y \leq r-x} \bar{\Pi}^{+}(r-y)+\int_{-r+x<y \leq r} \bar{\Pi}^{-}(r+y)\right) U_{r}(\mathrm{~d} y) \\
& +\bar{\Pi}^{+}(x) U_{r}((r-x, r])+\bar{\Pi}^{-}(x) U_{r}([-r,-r+x])
\end{aligned}
$$

while for $x \geq 2 r$

$$
P(|\Delta X(T(r))|>x)=\bar{\Pi}(x) U_{r}([-r, r]) .
$$

Proof of Lemma 1.4: The following joint and marginal distributions are in Lemma 2 and Corollary 2 of [7]:

$$
P(X(T(r)-) \in \mathrm{d} y, X(T(r)) \in \mathrm{d} z)=U_{r}(\mathrm{~d} y) \Pi(\mathrm{d} z-y), \text { for } 0 \leq|y| \leq r<|z|
$$


and

$$
P(X(T(r)) \in \mathrm{d} z)=\int_{|y| \leq r} \Pi(\mathrm{d} z-y) U_{r}(\mathrm{~d} y), \text { for }|z|>r .
$$

From (1.30) we obtain by integration

$$
P(X(T(r)-) \in \mathrm{d} y)=\left(\bar{\Pi}^{+}(r-y)+\bar{\Pi}^{-}(r+y)\right) U_{r}(\mathrm{~d} y), \text { for }|y|<r .
$$

Using these we obtain (1.26)-(1.29) as follows.

Parts (i), (ii): Equations (1.26) and (1.27) follow by integrating (1.31) and (1.32).

Part (iii): (1.28) and (1.29) are established in the proof of Lemma 6 of [7].

As an application of Lemma 1.4 we have:

Lemma 1.5. Suppose $\bar{\Pi}(0+)>0$ and $r>0$. Then for $0<c<d$ :

$$
P\left\{\left|\frac{\Delta X(T(r))}{r}\right| \in(c, d)\right\} \leq(\bar{\Pi}(r c)-\bar{\Pi}(r d)) U_{r}([-r, r]), 0<c<d .
$$

Proof of Lemma 1.5: This follows from (1.28) and (1.29) by checking cases, $0<c<d \leq$ $2,0<c \leq 2<d$, and $2<c<d$. For $0<c<d \leq 2$, we get from (1.28)

$$
\begin{aligned}
& P\left\{\left|\frac{\Delta X(T(r))}{r}\right| \in(c, d)\right\} \\
& =\left(\int_{r-r d<y \leq r-r c} \bar{\Pi}^{+}(r-y)+\int_{-r+r c<y \leq-r+r d} \bar{\Pi}^{-}(r+y)\right) U_{r}(\mathrm{~d} y) \\
& \quad+\bar{\Pi}^{+}(r c) U_{r}((r-r c, r])+\bar{\Pi}^{-}(r c) U_{r}([-r,-r+r c]) \\
& \quad-\bar{\Pi}^{+}(r d) U_{r}((r-r d, r])-\bar{\Pi}^{-}(r d) U_{r}([-r,-r+r d])
\end{aligned}
$$

Using upper bounds for $\bar{\Pi}^{ \pm}$we can bound this by

$$
\begin{aligned}
& \bar{\Pi}^{+}(r c) U_{r}((r-r d, r-r c])+\bar{\Pi}^{-}(r c) U_{r}((-r+r c,-r+r d]) \\
& +\bar{\Pi}^{+}(r c) U_{r}((r-r c, r])+\bar{\Pi}^{-}(r c) U_{r}([-r,-r+r c]) \\
& -\bar{\Pi}^{+}(r d) U_{r}((r-r d, r])-\bar{\Pi}^{-}(r d) U_{r}([-r,-r+r d]) \\
= & \bar{\Pi}^{+}(r c) U_{r}((r-r d, r])-\bar{\Pi}^{+}(r d) U_{r}((r-r d, r]) \\
& +\bar{\Pi}^{-}(r c) U_{r}([-r,-r+r d])-\bar{\Pi}^{-}(r d) U_{r}([-r,-r+r d]) \\
= & \left(\bar{\Pi}^{+}(r c)-\bar{\Pi}^{+}(r d)\right) U_{r}((r-r d, r])+\left(\bar{\Pi}^{-}(r c)-\bar{\Pi}^{-}(r d)\right) U_{r}([-r,-r+r d]) .
\end{aligned}
$$

Since $U_{r}(\cdot)$ is a measure, this is no bigger than

$$
\begin{aligned}
& \left(\bar{\Pi}^{+}(r c)-\bar{\Pi}^{+}(r d)\right) U_{r}([-r, r])+\left(\bar{\Pi}^{-}(r c)-\bar{\Pi}^{-}(r d)\right) U_{r}([-r, r]) \\
& =(\bar{\Pi}(r c)-\bar{\Pi}(r d)) U_{r}([-r, r]),
\end{aligned}
$$

as required for (1.33). The other two cases are similar, but easier, since we just subtract $\bar{\Pi}(r d) U_{r}([-r, r])$ for the " $d$ " part.

Lemma 1.6. Suppose $\bar{\Pi}(0+)>0$ and $r>0$, and $c_{2}, C_{2}$, are as in (1.22). Then we have the following inequalities.

(i) For $s>1$

$$
\frac{c_{2} \bar{\Pi}((s+1) r)}{h(r)} \leq P\left(\frac{|X(T(r))|}{r}>s\right) \leq \frac{C_{2} \bar{\Pi}((s-1) r)}{h(r)} .
$$

(ii) For $0<s<1$

$$
\frac{c_{2} \bar{\Pi}((1+s) r)}{h(r s)} \leq P\left(\frac{|X(T(r)-)|}{r} \leq s\right) \leq \frac{C_{2} \bar{\Pi}((1-s) r)}{h(r)} .
$$


(iii) When $x>0$

$$
\frac{c_{2} \bar{\Pi}(r \max (x, 2))}{h(r)} \leq P\left(\frac{|\Delta X(T(r))|}{r}>x\right) \leq \frac{C_{2} \bar{\Pi}(x r)}{h(r)} .
$$

Proof of Lemma 1.6: Parts (i) and (iii): (1.34) and the upper bound in (1.36) follow from Corollary 4 and Lemma 6 of [7], on noting the bounds in (1.22) for ET $r$ ). For the lower bound in (1.36), use (1.28) to deduce, for $0<x \leq 2$,

$$
\begin{aligned}
P(|\Delta X(T(r))|>x r) & \geq \bar{\Pi}^{+}(2 r) U_{r}\left((r[-1,1-x])+\bar{\Pi}^{+}(x r) U_{r}(r(1-x, 1])\right. \\
& +\bar{\Pi}^{-}(2 r) U_{r}(r[-1+x, 1])+\bar{\Pi}^{-}(x r) U_{r}(r[-1,-1+x]) \\
& \geq \bar{\Pi}^{+}(2 r) U_{r}(r[-1,1])+\bar{\Pi}^{-}(2 r) U_{r}(r[-1,1])=\bar{\Pi}(2 r) U_{r}(r[-1,1]) .
\end{aligned}
$$

Thus, using this together with (1.29), we have for $x>0$,

$$
P(|\Delta X(T(r))|>x r) \geq \bar{\Pi}(r \max (x, 2)) U_{r}(r[-1,1]),
$$

and use of (1.25) and (1.22) gives the lower bound in (1.36).

Part (ii): From (1.25) and (1.27), for $0<s<1$,

$$
\begin{gathered}
P(|X(T(r)-)| \leq r s) \leq\left(\bar{\Pi}^{+}(r-r s)+\bar{\Pi}^{-}(r-r s)\right) U_{r}([-r, r]) \\
=\bar{\Pi}((1-s) r) U_{r}([-r s, r s]) \leq \bar{\Pi}((1-s) r) U_{r}([-r, r]),
\end{gathered}
$$

and, in the other direction,

$$
\begin{gathered}
P(|X(T(r)-)| \leq r s) \geq\left(\bar{\Pi}^{+}\left((r+r s)+\bar{\Pi}^{-}(r+r s)\right) U_{r}([-r s, r s])\right. \\
=\bar{\Pi}((1+s) r) U_{r}([-r s, r s]) \geq \bar{\Pi}((1+s) r) U_{r s}([-r s, r s]) .
\end{gathered}
$$

Then use (1.22) again.

See [11], [12] for random walk versions of many of the above results.

The next lemma provides another useful bound.

Lemma 1.7. Assume $\bar{\Pi}(0+)=\infty$ and $X(t) \in F C$ at 0 . Recall the function $U(\cdot)$ defined in (1.4), and set

$$
t(r):=r^{2} / U(r), r>0 .
$$

Then there is an $r_{0}>0$ and a finite constant $C>0$ such that for $0<r \leq r_{0}, 0 \leq c<d<1$ and $\varepsilon \in(0,1)$

$$
\begin{gathered}
\mathbf{P}\left\{\frac{|X(T(r)-)|}{r} \in[c, d)\right\} \leq \\
\frac{2 C}{(1-d)^{2}}\left(\left(1+128 c_{1}^{2}\right) \varepsilon+\int_{\varepsilon}^{\varepsilon^{-1}} \mathbf{P}\left\{c \leq \frac{1}{r}|X(s t(r))|<d\right\} \mathrm{d} s\right),
\end{gathered}
$$

where $c_{1}$ is the constant in (1.21).

Proof of Lemma 1.7: Take constants $0<c<d<1$. From (1.27) we get

$$
\begin{aligned}
& \mathbf{P}\left\{\frac{|X(T(r)-)|}{r} \in[c, d)\right\}=\int_{c \leq|y|<d}\left(\bar{\Pi}^{+}(r(1-y))+\bar{\Pi}^{-}(r(1+y))\right) U_{r}(r \mathrm{~d} y) \\
& =\left(\int_{-d<y \leq-c}+\int_{c \leq y<d}\right)\left(\bar{\Pi}^{+}(r(1-y))+\bar{\Pi}^{-}(r(1+y))\right) U_{r}(r \mathrm{~d} y) .
\end{aligned}
$$

With elementary estimates we can bound the RHS by the quantities

$$
\left(\bar{\Pi}^{+}(r(1+c))+\bar{\Pi}^{-}(r(1-d))\right) U_{r}(r(-d,-c])+\left(\bar{\Pi}^{+}(r(1-d))+\bar{\Pi}^{-}(r(1+c))\right) U_{r}(r[c, d))
$$


Compactness and continuity properties for two-sided Lévy exit times

$$
\leq t(r)(\bar{\Pi}(r(1-d))+\bar{\Pi}(r(1+c))) \frac{1}{t(r)}\left(U_{r}(r(-d,-c])+U_{r}(r[c, d))\right) .
$$

Notice that

$$
\begin{aligned}
t(r)(\bar{\Pi}(r(1-d))+ & \bar{\Pi}(r(1+c)))=\frac{r^{2}}{U(r)}(\bar{\Pi}(r(1-d))+\bar{\Pi}(r(1+c))) \\
\leq & \frac{r^{2} \bar{\Pi}(r(1-d))}{U(r(1-d))}+\frac{r^{2} \bar{\Pi}(r)}{U(r)}
\end{aligned}
$$

Since $X(t) \in F C$ at 0 , by (1.14) with $V$ replaced by $U$ we can find a finite constant $C>0$ such that for $r \leq$ some $r_{0}>0$ ( $r_{0}$ not depending on $c$ or $d$ ) the RHS of (1.40) does not exceed

$$
C\left(\frac{1}{(1-d)^{2}}+1\right) \leq \frac{2 C}{(1-d)^{2}} .
$$

For the other term in (1.39), by changing variable to $t=t(r) s$ and noting that

$$
\sup _{0 \leq u<t(r) s}|X(u)|=\sup _{0 \leq v<s}|X(t(r) v)|,
$$

we obtain from (1.24)

$$
\begin{gathered}
\frac{1}{t(r)}\left(U_{r}(r(-d,-c])+U_{r}(r[c, d))\right) \\
=\int_{0}^{\infty} \mathbf{P}\left\{\sup _{0 \leq v<s}|X(t(r) v)| \leq r,-r d<X(s t(r)-) \leq-r c \text { or } r c \leq X(s t(r)-)<r d\right\} \mathrm{d} s \\
=\int_{0}^{\infty} \mathbf{P}\left\{\sup _{0 \leq v<s}|X(t(r) v)| \leq r, r c \leq|X(s t(r)-)|<r d\right\} \mathrm{d} s .
\end{gathered}
$$

Now for $s>0$

$$
\begin{gathered}
\mathbf{P}\left\{\sup _{0 \leq v<s}|X(t(r) v)| \leq r\right\} \\
\leq \mathbf{P}\left\{\sup _{0 \leq v<s / 2}|X(t(r) v)| \leq r, \sup _{s / 2 \leq v<s}|X(t(r) v)-X(t(r) s / 2)| \leq 2 r\right\} \\
\leq \mathbf{P}^{2}\left\{\sup _{0 \leq v<s / 2}|X(t(r) v)| \leq 2 r\right\}=\mathbf{P}^{2}\left\{\sup _{0 \leq v<t(r) s / 2}|X(v)| \leq 2 r\right\} .
\end{gathered}
$$

Then by (1.21), (1.5) and (1.6) we obtain, for all $s>0$ and $r>0$,

$$
\mathbf{P}^{2}\left\{\sup _{0 \leq v<t(r) s / 2}|X(v)| \leq 2 r\right\} \leq \frac{16 c_{1}^{2}}{t^{2}(r) s^{2} h^{2}(2 r)}=\frac{16 c_{1}^{2} U^{2}(r)}{r^{4} s^{2} h^{2}(2 r)} \leq \frac{128 c_{1}^{2} U^{2}(r)}{r^{4} s^{2} h^{2}(r)} \leq \frac{128 c_{1}^{2}}{s^{2}} .
$$

Then from (1.39), (1.40), (1.41) and (1.42) we can observe that, for any $0<\varepsilon<1$ and $0<r \leq r_{0}$,

$$
\begin{gathered}
\mathbf{P}\left\{\frac{|X(T(r)-)|}{r} \in[c, d)\right\} \\
\leq \frac{2 C}{(1-d)^{2}}\left(\varepsilon+\int_{\varepsilon}^{\varepsilon^{-1}} \mathbf{P}\left\{c \leq \frac{1}{r}|X(s t(r)-)|<d\right\} \mathrm{d} s+\int_{\varepsilon^{-1}}^{\infty} \frac{128 c_{1}^{2}}{s^{2}} \mathrm{~d} s\right) .
\end{gathered}
$$

Since, with probability $1, X$ does not jump at any given point in $(0, \infty)$, the RHS is equal to the RHS of (1.38). 
When $c=0<d<1$ replace the RHS of (1.39) by

$$
\int_{-d<y<d}\left(\bar{\Pi}^{+}(r(1-y))+\bar{\Pi}^{-}(r(1+y))\right) U_{r}(r \mathrm{~d} y) \leq \bar{\Pi}(r(1-d)) U_{r}(r(-d, d)),
$$

and note that (1.41) remains true with the smaller quantity

$$
\frac{r^{2} \bar{\Pi}(r(1-d))}{U(r(1-d))} \leq \frac{C}{(1-d)^{2}}
$$

on the RHS. Thus (1.43) remains true in this case too and we get (1.38) again.

\subsection{Subsequential relative stability of $X(T(r)) / r$ and $X(T(r)-) / r$}

The next theorem gives equivalences for the subsequential relative stability of $|X(T(r))| / r$ and $|X(T(r)-)| / r$ at 0 , by which we mean the existence of a non-stochastic sequence $r_{k} \downarrow 0$ such that (1.44) or (1.46) hold.

Theorem 1.8. Suppose $\bar{\Pi}(0+)>0$. The following are all equivalent:

(a)(i) there is a non-stochastic sequence $r_{k} \downarrow 0$ as $k \rightarrow \infty$ for which

$$
\frac{\left|X\left(T\left(r_{k}\right)\right)\right|}{r_{k}} \stackrel{P}{\rightarrow} 1 \text { as } k \rightarrow \infty
$$

(a) (ii) there is a non-stochastic sequence $r_{k} \downarrow 0$ and a constant $c>1$ for which

$$
P\left(\frac{\left|X\left(T\left(r_{k}\right)\right)\right|}{r_{k}} \leq c\right) \rightarrow 1 \text { as } k \rightarrow \infty
$$

(b)(i) there is a non-stochastic sequence $r_{k} \downarrow 0$ for which

$$
\frac{\left|X\left(T\left(r_{k}\right)-\right)\right|}{r_{k}} \stackrel{P}{\rightarrow} 1 \text { as } k \rightarrow \infty
$$

(b)(ii) there is a non-stochastic sequence $r_{k} \downarrow 0$ and a constant $c<1$ for which

$$
P\left(\frac{\left|X\left(T\left(r_{k}\right)-\right)\right|}{r_{k}} \leq c\right) \rightarrow 0 \text { as } k \rightarrow \infty
$$

(c) there is a non-stochastic sequence $r_{k} \downarrow 0$ such that for some (hence every) $c>0$

$$
P\left(\frac{\left|\Delta X\left(T\left(r_{k}\right)\right)\right|}{r_{k}}>c\right) \rightarrow 0, \text { as } k \rightarrow \infty
$$

(d)

$$
\limsup _{x \downarrow 0} \frac{x|\nu(x)|+U(x)}{x^{2} \bar{\Pi}(x)}=\infty .
$$

The following corollary is an immediate consequence of Theorem 1.8.

Corollary 1.9. (i) If, for some $r_{k} \downarrow 0$, as $k \rightarrow \infty$,

$$
\frac{\left|X\left(T\left(r_{k}\right)\right)\right|}{r_{k}} \stackrel{P}{\rightarrow} a \text {, for some } a \in[1, \infty), \text { then } \frac{\left|X\left(T\left(r_{k}\right)\right)\right|}{r_{k}} \stackrel{P}{\rightarrow} 1 ;
$$

(ii) if, for some $r_{k} \downarrow 0$, as $k \rightarrow \infty$,

$$
\frac{\left|X\left(T\left(r_{k}\right)-\right)\right|}{r_{k}} \stackrel{P}{\rightarrow} a \text {, for some } a \in(0,1], \text { then } \frac{\left|X\left(T\left(r_{k}\right)-\right)\right|}{r_{k}} \stackrel{P}{\rightarrow} 1 .
$$


Proof of Theorem 1.8: Suppose $\bar{\Pi}(0+)>0$ throughout.

(d) $\Longrightarrow(\mathrm{b})(\mathrm{i}) \Longrightarrow(\mathrm{b})(\mathrm{ii}) \Longrightarrow(\mathrm{d})$ : We shall provide proofs of this sequence of equivalences; the proof of (d) $\Longrightarrow$ (a)(i) $\Longrightarrow$ (a)(ii) $\Longrightarrow$ (d) is similar. Assume (1.49) holds, or, equivalently,

$$
\liminf _{x \downarrow 0} \bar{\Pi}(x) / h(x)=0 .
$$

For all $0<s<1$ and $r>0$ we get by (1.35) and (1.6)

$$
P\left(\frac{|X(T(r)-)|}{r} \leq s\right) \leq \frac{C_{2} \bar{\Pi}(r(1-s))}{h(r)} \leq \frac{3 C_{2} \bar{\Pi}(r(1-s))}{h(r(1-s))} .
$$

By (1.52) there is a decreasing sequence $x_{k} \downarrow 0$ such that

$$
\lim _{k \rightarrow \infty} \bar{\Pi}\left(x_{k}\right) / h\left(x_{k}\right)=0 .
$$

Thus, by (1.53) the sequence $y_{k}(s):=x_{k} /(1-s)$ satisfies, for all $0<s<1$,

$$
P\left\{\left|\frac{X\left(T\left(y_{k}(s)-\right)\right.}{y_{k}(s)}\right| \leq s\right\} \rightarrow 0, \text { as } k \rightarrow \infty .
$$

Choose $k_{1} \geq 1$ so that

$$
P\left\{\left|\frac{X\left(T\left(y_{k_{1}}(1 / 2)-\right)\right)}{y_{k_{1}}(1 / 2)}\right| \leq 1-\frac{1}{2}\right\} \leq \frac{1}{2},
$$

and, for $j \geq 2$, choose $k_{j}>k_{j-1}$ so that $y_{k_{j}}\left(1-2^{-j}\right)<y_{k_{j-1}}\left(1-2^{-j+1}\right)$ and

$$
P\left\{\left|\frac{X\left(T\left(y_{k_{j}}\left(1-2^{-j}\right)-\right)\right)}{y_{k_{j}}\left(1-2^{-j}\right)}\right| \leq 1-\frac{1}{2^{j}}\right\} \leq \frac{1}{2^{j}} .
$$

Thus we see that for the sequence $z_{j}=y_{k_{j}}\left(1-2^{-j}\right) \downarrow 0$ we have

$$
\frac{X\left(T\left(z_{j}\right)-\right)}{z_{j}} \stackrel{P}{\rightarrow} 1 .
$$

This implies (1.46) and clearly (1.46) implies (1.47).

Now suppose (1.47) is true for some $0<c<1$ and sequence $r_{k} \downarrow 0$, and we show that (1.49) holds. By (1.35) and (1.6)

$$
P\left\{\left|\frac{X\left(T\left(r_{k}\right)-\right)}{r_{k}}\right| \leq c\right\} \geq \frac{c_{2} \bar{\Pi}\left((1+c) r_{k}\right)}{h\left(c r_{k}\right)} \geq \frac{c_{2} c^{2} \bar{\Pi}\left((1+c) r_{k}\right)}{2(1+c)^{2} h\left((1+c) r_{k}\right)} .
$$

By (1.47), the LHS tends to 0 , thus (1.49) holds via (1.52). So we have shown (d) $\Longrightarrow$ (b)(i) $\Longrightarrow$ (b)(ii) $\Longrightarrow$ (d).

(c) $\Longleftrightarrow$ (d): Suppose (1.48) holds for $r_{k} \downarrow 0$ and some $c>0$. Then by (1.36) and (1.6), (1.54) holds with $x_{k}$ replaced by $r_{k}$. Thus (1.49) holds via (1.52).

Conversely, assume (1.49) and hence that (1.54) holds for an $x_{k} \downarrow 0$. Take any $\varepsilon \in(0,1)$ and let $y_{k}(\varepsilon):=x_{k} / \varepsilon$. Then (1.54) and (1.6) together imply $\lim _{k \rightarrow \infty} \bar{\Pi}\left(\varepsilon y_{k}(\varepsilon)\right) / h\left(y_{k}(\varepsilon)\right)=$ 0 . Then by (1.36),

$$
\lim _{k \rightarrow \infty} P\left(\frac{\left|\Delta X\left(T\left(y_{k}(\varepsilon)\right)\right)\right|}{y_{k}(\varepsilon)}>\varepsilon\right)=0 .
$$

Take sequences $\varepsilon_{\ell} \downarrow 0$ and $\delta_{\ell} \downarrow 0$ as $\ell \rightarrow \infty$. Then by (1.55), we can make

$$
P\left(\frac{\left|\Delta X\left(T\left(y_{k}\left(\varepsilon_{\ell}\right)\right)\right)\right|}{y_{k}\left(\varepsilon_{\ell}\right)}>\varepsilon_{\ell}\right) \leq \delta_{\ell}
$$


for all $k \geq$ some $k_{0}(\ell)$. Let $z_{\ell}:=y_{k_{0}(\ell)}\left(\varepsilon_{\ell}\right)$. Given arbitrary $\varepsilon>0, \delta>0$, choose $\ell_{0}=\ell_{0}(\varepsilon, \delta)$ so large that $\varepsilon_{\ell} \leq \varepsilon$ and $\delta_{\ell} \leq \delta$ whenever $\ell \geq \ell_{0}$. Then by (1.56)

$$
P\left(\frac{\left|\Delta X\left(T\left(z_{\ell}\right)\right)\right|}{z_{\ell}}>\varepsilon\right) \leq \delta
$$

for all $\ell \geq \ell_{0}$. This proves that (1.48) holds for any $c>0$. Hence we have shown (c) $\Longleftrightarrow$ (d).

\section{The position before exit}

In this section we confine attention to $X(T(r)-)$.

\subsection{Stochastic compactness of $|X(T(r)-)| / r$ at $\mathbf{0}$}

We say that $|X(T(r)-)| / r$ is stochastically compact at 0 , written $|X(T(r)-)| / r$ is in $S C$ at 0 , if every sequence $r_{k} \downarrow 0$ has a subsequence $r_{k^{\prime}} \downarrow 0$ such that

$$
\frac{\left|X\left(T\left(r_{k^{\prime}}\right)-\right)\right|}{r_{k^{\prime}}} \stackrel{\mathrm{D}}{\rightarrow} W^{\prime},
$$

where $W^{\prime}$ is a finite nondegenerate random variable which may depend on the choice of subsequence $r_{k^{\prime}}$.

Theorem 2.1. Assume $\bar{\Pi}(0+)=\infty$. Then $|X(T(r)-)| / r$ is in $S C$ at 0 if and only if

$$
\limsup _{x \downarrow 0} \frac{x|\nu(x)|+U(x)}{x^{2} \bar{\Pi}(x)}<\infty
$$

and

$$
\text { for no sequence } r_{k} \downarrow 0 \text { does } \frac{\left|X\left(T\left(r_{k}\right)-\right)\right|}{r_{k}} \stackrel{\mathrm{P}}{\rightarrow} 0 \text {, as } k \rightarrow \infty \text {. }
$$

Remark 2.2. The type of convergence in (2.2) can occur. Suppose $X(t)$ is a subordinator with Lévy measure $\Pi$. Then according to Thm. 6, p.81, of [2], and the remarks following it, $X(T(r)-) / r \stackrel{\mathrm{P}}{\rightarrow} 0$ as $r \downarrow 0$ if and only if the Laplace exponent of $X$ is slowly varying at infinity. This is the case for example with the gamma process $X(t)$, having Lévy measure $\Pi(\mathrm{d} x)=a x^{-1} e^{-b x} \mathbf{1}\{x>0\}$, for some $a>0, b>0$.

Proof of Theorem 2.1: Since $|X(T(r)-)| / r \leq 1$ is always relatively compact (equivalently, tight), $|X(T(r)-)| / r$ is in $S C$ at 0 iff there can be no subsequence $r_{k} \downarrow 0$ such that $\left|X\left(T\left(r_{k}\right)-\right)\right| / r_{k} \stackrel{P}{\rightarrow} a$, with $a \in[0,1]$. When $X$ satisfies (2.1) the possibility $a \in(0,1]$ is impossible by Theorem 1.8 and Corollary 1.9 , while the possibility $a=0$ is ruled out because it would contradict (2.2).

Corollary 2.3. Assume $\bar{\Pi}(0+)=\infty$ and $X(t) \in F C_{0}$ at 0 , but $X(t) \notin D_{P}(N)$ at 0 . Then $|X(T(r)-)| / r$ is in $S C$ at 0.

Proof of Corollary 2.3: Assume $\bar{\Pi}(0+)=\infty$ and $X(t) \in F C_{0}$ at 0 , but $X(t) \notin D_{P}(N)$ at 0 . Recall by Theorem 1.1 that $X(t) \in F C_{0}$ at 0 holds if and only if

$$
\limsup _{x \downarrow 0}\left(x^{2} \bar{\Pi}(x)+x|\nu(x)|\right) / V(x)<\infty
$$

and by Theorem 1.3, $X(t) \notin D_{P}(N)$ at 0 holds if and only if

$$
\liminf _{x \downarrow 0} x^{2} \bar{\Pi}(x) / V(x)>0 .
$$


Thus (2.1) is satisfied. Now $|X(T(r)-)| / r \leq 1$ is always relatively compact. Take any $r_{k} \downarrow 0$ such that $\left|X\left(T\left(r_{k}\right)-\right)\right| / r_{k} \stackrel{\mathrm{D}}{\longrightarrow} W$ for a finite rv $W$. Since (2.1) holds, $W$ cannot be degenerate at a positive constant by Theorem 1.8.

This rules out the possibility of a nonzero degenerate limit for $\left|X\left(T\left(r_{k}\right)-\right)\right| / r_{k}$ but there is still the possibility of a zero limit. Suppose by way of contradiction $W=0$ a.s. This is impossible, as follows. Since $X(t) \in F C_{0}$ at 0 implies $X(t) \in F C$ at 0 , we can apply Lemma 1.7, in which we take $c=0$, replace $r$ by $r_{k}$ and replace $t(r)$ by $t_{k}=t\left(r_{k}\right)$. Then from (1.38), for $k$ large enough, and any $d \in(0,1)$ and $\varepsilon \in(0,1)$,

$$
\mathbf{P}\left\{\frac{\left|X\left(T\left(r_{k}\right)-\right)\right|}{r_{k}}<d\right\} \leq \frac{2 C}{(1-d)^{2}}\left(\left(1+128 c_{1}^{2}\right) \varepsilon+\int_{\varepsilon}^{\varepsilon^{-1}} \mathbf{P}\left\{\frac{1}{r_{k}}\left|X\left(s t_{k}\right)\right|<d\right\} \mathrm{d} s\right) .
$$

Notice that $t\left(r_{k}\right)$ satisfying (1.37) with $r=r_{k}$ implies $r_{k}=b\left(t_{k}\right)$, where $b(t)$ is the unique inverse to the function $x \mapsto x^{2} / U(x)$; see (1.17). Since $X(t) \in F C_{0}$ at 0 we have the functional convergence in (1.16). So, by taking a further subsequence of $t_{k}$ if necessary, there is a process $\left(Y^{\prime}(s)\right)$ such that

$$
\lim _{k \rightarrow \infty} \mathbf{P}\left\{\frac{1}{r_{k}}\left|X\left(s t_{k}\right)\right|<d\right\}=\lim _{k \rightarrow \infty} \mathbf{P}\left\{\frac{1}{b\left(t_{k}\right)}\left|X\left(s t_{k}\right)\right|<d\right\}=\mathbf{P}\left\{\left|Y^{\prime}(s)\right|<d\right\}
$$

at points of continuity $d>0$ of the distribution of $Y^{\prime}(s)$. As pointed out in Remark 1.2, any subsequential limit rv such as $Y^{\prime}(s)$ has an absolutely continuous, hence continuous, distribution for each $s>0$. By dominated convergence the integral on the RHS of (2.3) has limit $\int_{\varepsilon}^{\varepsilon^{-1}} \mathbf{P}\left\{0 \leq\left|Y^{\prime}(s)\right|<d\right\} \mathrm{d} s$ as $k \rightarrow \infty$. Since $d$ and $\varepsilon$ can be taken arbitrarily small, (2.3) shows that $\left|X\left(T\left(r_{k}\right)-\right)\right| / r_{k} \stackrel{\mathrm{P}}{\rightarrow} 0$ is impossible and again we have a contradiction.

\subsection{Continuity of subsequential limit distributions, $|X(T(r)-)| / r$}

In this subsection we give conditions under which any subsequential limit rv of a stochastically compact $|X(T(r)-)| / r$ has a continuous distribution.

Theorem 2.4. Assume $\bar{\Pi}(0+)=\infty$ and $X(t) \in F C_{0}$ at 0 . Assume also $X(t) \notin D_{P}(N)$ at 0 . Then by Corollary 2.3, $|X(T(r)-)| / r$ is in $S C$ at 0 . Take any sequence $r_{k} \downarrow 0$ such that

$$
\frac{\left|X\left(T\left(r_{k}\right)-\right)\right|}{r_{k}} \stackrel{\mathrm{D}}{\rightarrow} W,
$$

where $W$ is a finite nondegenerate random variable. Then the distribution of $W$ is continuous at every point in $(0,1)$.

Proof of Theorem 2.4: Assume $\bar{\Pi}(0+)=\infty$ and $X(t) \in F C_{0}$ at 0 , and that (2.4) holds for a given sequence $r_{k} \downarrow 0$. Let $t_{k}=t\left(r_{k}\right)$, where $t(r)$ is defined in (1.37), so that $r_{k}=b\left(t_{k}\right)$, and choose $0<c<d<1$. Then by (1.38), for any $\varepsilon>0$ and large $k$,

$$
\begin{gathered}
\mathbf{P}\left\{\frac{\left|X\left(T\left(r_{k}\right)-\right)\right|}{r_{k}} \in[c, d)\right\} \\
\leq \frac{2 C}{(1-d)^{2}}\left(\left(1+128 c_{1}^{2}\right) \varepsilon+\int_{\varepsilon}^{\varepsilon^{-1}} \mathbf{P}\left\{c \leq \frac{1}{r_{k}}\left|X\left(s t_{k}\right)\right|<d\right\} \mathrm{d} s\right) .
\end{gathered}
$$

By (1.16), taking a further subsequence of $t_{k}$ if necessary, we can make

$$
\mathbf{P}\left\{c<\frac{1}{r_{k}}\left|X\left(s t_{k}\right)\right|<d\right\} \rightarrow \mathbf{P}\left\{c<\left|Y^{\prime}(s)\right|<d\right\}, \text { as } k \rightarrow \infty,
$$


where by Remark 1.2 the Lévy process $Y^{\prime}(s)$ has a continuous distribution for each $s>0$. Therefore by the bounded convergence theorem

$$
\lim _{k \rightarrow \infty} \int_{\varepsilon}^{\varepsilon^{-1}} \mathbf{P}\left\{c<\frac{1}{r_{k}}\left|X\left(s t_{k}\right)\right|<d\right\} \mathrm{d} s=\int_{\varepsilon}^{\varepsilon^{-1}} \mathbf{P}\left\{c<\left|Y^{\prime}(s)\right|<d\right\} \mathrm{d} s,
$$

for every $0<\varepsilon<1$, and, again by the bounded convergence theorem,

$$
\int_{\varepsilon}^{\varepsilon^{-1}} \mathbf{P}\left\{b-1 / m<\left|Y^{\prime}(s)\right|<b+1 / m\right\} \mathrm{d} s \rightarrow 0, \text { as } m \rightarrow \infty,
$$

for any $b \in(0,1)$. Substituting $c=b-1 / m$ and $d=b+1 / m$, with $d+1 / m<1$ and $1 / m<b$, in (2.5), implies that for all $\varepsilon>0$ there exists an $m_{0}=m_{0}(\varepsilon)>1$ such that for all $m \geq m_{0}$

$$
\limsup _{k \rightarrow \infty} \mathbf{P}\left\{\frac{\left|X\left(T\left(r_{k}\right)-\right)\right|}{r_{k}} \in(b-1 / m, b+1 / m)\right\} \leq \frac{4 C}{(1-b-1 / m)^{2}}\left(1+128 c_{1}^{2}\right) \varepsilon .
$$

Now we argue as follows. By (2.4) and the Portmanteau theorem ([4], p.15)

$$
\mathbf{P}\{W \in(b-1 / m, b+1 / m)\} \leq \liminf _{k \rightarrow \infty} \mathbf{P}\left\{\frac{\left|X\left(T\left(r_{k}\right)-\right)\right|}{r_{k}} \in(b-1 / m, b+1 / m)\right\} .
$$

For any $m \geq m_{0}(\varepsilon)$ the RHS here does not exceed

$$
\limsup _{k \rightarrow \infty} \mathbf{P}\left\{\frac{\left|X\left(T\left(r_{k}\right)-\right)\right|}{r_{k}} \in(b-1 / m, b+1 / m)\right\},
$$

which by (2.6) does not exceed $4 C\left(1+128 c_{1}^{2}\right) \varepsilon /(1-b-1 / m)^{2}$. Since $\varepsilon>0$ can be made arbitrarily small, this implies that the distribution of $W$ is continuous at $b$.

\section{The position after exit}

In this section we confine attention to $X(T(r))$.

\subsection{Stochastic compactness of $|X(T(r))| / r$ at $\mathbf{0}$}

We say that $|X(T(r))| / r$ is in $S C$ at 0 if every sequence $r_{k} \downarrow 0$ has a subsequence $\left\{r_{k^{\prime}}\right\}$ such that $\left|X\left(T\left(r_{k^{\prime}}\right)\right)\right| / r_{k^{\prime}}$ converges in distribution to a finite nondegenerate random variable.

In the next theorem, Part (i) is from Theorem 2 of [7].

Theorem 3.1. Assume $\bar{\Pi}(0+)=\infty$. (i) $|X(T(r))| / r$ is tight as $r \downarrow 0$ if and only if

$$
\lim _{\lambda \rightarrow \infty} \limsup _{x \downarrow 0} \frac{x^{2} \bar{\Pi}(x \lambda)}{x|\nu(x)|+U(x)}=0 .
$$

(ii) $|X(T(r))| / r$ is in $S C$ at 0 if and only if (2.1) and (3.1) hold;

(iii) furthermore (2.1) and (3.1) hold if and only if $X(t) \in F C_{0} \backslash D_{P}(N)$ at 0 .

Remark 3.2. Regarding Part (i) of Theorem 3.1: Condition (3.1) by itself characterizes yet another stochastic compactness class for the process $X$, which we might term $F C^{\prime}$. Specifically, we say that $X(t) \in F C^{\prime}$ at 0 if there exists a non-stochastic function $b(t)>0$ such that each sequence $t_{k} \downarrow 0$ contains a subsequence $t_{k^{\prime}} \downarrow 0$ for which

$$
\frac{X\left(t_{k^{\prime}}\right)}{b\left(t_{k^{\prime}}\right)} \stackrel{\mathrm{D}}{\longrightarrow} Y^{\prime}, \text { as } t_{k^{\prime}} \downarrow 0
$$

where $Y^{\prime}$ is an a.s. finite random variable which may be degenerate at a constant, but is not degenerate at 0 . We omit the proof and further discussion of this here. Class $F C^{\prime}$ is analogous to a class $S C^{\prime}$ introduced by [10] which characterizes a similar property for random walks. 
Proof of Theorem 3.1: Proof of Part (i): see Theorem 2 of [7]. The proof follows by an easy argument based on inequalities (1.6) and (1.34).

Proof of Part (ii). Assume that $|X(T(r))| / r$ is in $S C$ at zero. Then $|X(T(r))| / r$ is tight as $r \downarrow 0$ so (3.1) holds. Suppose that (2.1) does not hold. But then by Theorem 1.8, $\left|X\left(T\left(r_{k}\right)\right)\right| / r_{k} \stackrel{\mathrm{P}}{\rightarrow} 1$ for some $r_{k} \downarrow 0$ as $k \rightarrow \infty$, a contradiction.

Conversely, assume (3.1) and (2.1) are satisfied. By (3.1), $|X(T(r))| / r$ is tight as $r \downarrow 0$. Suppose there exists a constant $1 \leq a<\infty$ and a sequence $r_{k} \downarrow 0$ such that

$$
\frac{\left|X\left(T\left(r_{k}\right)\right)\right|}{r_{k}} \stackrel{P}{\rightarrow} a, \text { as } k \rightarrow \infty .
$$

Then by Corollary 1.9 and Theorem 1.8, (1.49) holds, which contradicts (2.1). Thus $|X(T(r))| / r$ is in $S C$ at 0 .

Proof of Part (iii). Assume (2.1) and (3.1). Now (2.1) implies the contrapositive of (1.19), so $X \notin D_{P}(N)$. Also (2.1) says that

$$
\limsup _{x \downarrow 0} \frac{h(x)}{\bar{\Pi}(x)}=\limsup _{x \downarrow 0} \frac{x|\nu(x)|+U(x)}{x^{2} \bar{\Pi}(x)}<\infty .
$$

To complete the proof of (1.15) we shall need the following fact motivated by a result of [6].

Fact A Assume $\bar{\Pi}(0+)=\infty$. A nondegenerate Lévy process $(X(t))_{t \geq 0}$ is in the Feller class at 0 whenever

$$
\lim _{\lambda \rightarrow \infty} \limsup _{x \downarrow 0} \frac{\bar{\Pi}(\lambda x)}{\bar{\Pi}(x)}<1 .
$$

(For a proof see the Appendix.)

Now take $\lambda>0$ and write

$$
\frac{\bar{\Pi}(\lambda x)}{\bar{\Pi}(x)}=\left(\frac{x^{2} \bar{\Pi}(\lambda x)}{x|\nu(x)|+U(x)}\right)\left(\frac{x|\nu(x)|+U(x)}{x^{2} \bar{\Pi}(x)}\right) .
$$

Then by (3.1) and (3.3)

$$
\lim _{\lambda \rightarrow \infty} \limsup _{x \downarrow 0} \frac{\bar{\Pi}(\lambda x)}{\bar{\Pi}(x)}=0 .
$$

It follows then from Fact $A$ and (1.14) that

$$
\limsup _{x \downarrow 0} \frac{x^{2} \bar{\Pi}(x)}{V(x)}<\infty,
$$

which together with (3.3) and $x^{2} \bar{\Pi}(x) \leq U(x)$ implies that

$$
\limsup _{x \downarrow 0} \frac{x^{2} \bar{\Pi}(x)+x|\nu(x)|}{V(x)} \leq \limsup _{x \downarrow 0}\left(\frac{U(x)+x|\nu(x)|}{x^{2} \bar{\Pi}(x)}\right)\left(\frac{x^{2} \bar{\Pi}(x)}{V(x)}\right)<\infty .
$$

So (1.15) is proved and hence $X(t) \in F C_{0}$ at 0 . Therefore $X(t) \in F C_{0} \backslash D_{P}(N)$ at 0 .

Going the other way, assume that $X(t) \in F C_{0} \backslash D_{P}(N)$ at 0 . First observe that $X(t) \in F C_{0}$ at 0 implies by (1.15) that

$$
\limsup _{x \downarrow 0} \frac{x^{2} \bar{\Pi}(x)+x|\nu(x)|}{V(x)}=: d<\infty,
$$

and from $X(t) \notin D_{P}(N)$ at 0 we get from (1.19) that

$$
\liminf _{x \downarrow 0} \frac{x^{2} \bar{\Pi}(x)}{V(x)}>0 .
$$


From these we infer that

$$
\begin{aligned}
\liminf _{x \downarrow 0} \frac{\bar{\Pi}(x)}{h(x)} & =\liminf _{x \downarrow 0} \frac{x^{2} \bar{\Pi}(x) / V(x)}{\left(x|\nu(x)|+x^{2} \bar{\Pi}(x)\right) / V(x)+1} \\
& \geq \liminf _{x \downarrow 0} \frac{x^{2} \bar{\Pi}(x) / V(x)}{d+1}>0 .
\end{aligned}
$$

Hence (2.1) holds. Next notice that for any $\lambda>1$

$$
\frac{x^{2} \bar{\Pi}(\lambda x)}{U(x)}=\frac{(\lambda x)^{2} \bar{\Pi}(\lambda x)}{U(\lambda x)}\left(\frac{U(\lambda x)}{U(x)}\right) \frac{1}{\lambda^{2}} .
$$

If $X(t) \in F C_{0}$, then necessarily $X(t) \in \mathrm{FC}$ at 0 . By arguing as on page 2219 of [15] we get for some $0<c_{1}<1$ and $K>0$ that for all small enough $x>0$

$$
\frac{x^{2} \bar{\Pi}(\lambda x)}{U(x)} \leq K \lambda^{2 c_{1}-2} .
$$

Since

we see from (3.6) that

$$
\frac{\bar{\Pi}(\lambda x)}{h(x)}=\frac{x^{2} \bar{\Pi}(\lambda x)}{x|\nu(x)|+U(x)} \leq \frac{x^{2} \bar{\Pi}(\lambda x)}{U(x)},
$$

$$
\lim _{\lambda \rightarrow \infty} \limsup _{x \downarrow 0} \frac{\bar{\Pi}(\lambda x)}{h(x)}=0,
$$

and thus (3.1) is also satisfied.

3.2 Continuity of subsequential limit distributions, $|X(T(r))| / r$

Lemma 3.3. Assume $\bar{\Pi}(0+)=\infty$. Suppose for a sequence $r_{k} \downarrow 0$

$$
\frac{\left|X\left(T\left(r_{k}\right)\right)\right|}{r_{k}} \stackrel{\mathrm{D}}{\longrightarrow} W
$$

where $W$ is a nondegenerate random variable. Suppose there exist nonnegative decreasing functions $\bar{\Lambda}^{+}$and $\bar{\Lambda}^{-}$on $(0, \infty)$, which vanish at infinity, and a sequence of positive constants $\left\{t_{k}\right\}_{k \geq 1}$, such that for all $u>0$ continuity points of $\Lambda^{ \pm}$,

$$
t_{k} \bar{\Pi}^{+}\left(r_{k} u\right) \rightarrow \bar{\Lambda}^{+}(u) \text { and } t_{k} \bar{\Pi}^{-}\left(r_{k} u\right) \rightarrow \bar{\Lambda}^{-}(u)
$$

and

$$
\limsup _{k \rightarrow \infty}\left(t_{k} h\left(r_{k}\right)\right)^{-1}=: \kappa<\infty .
$$

Suppose in addition that $\Lambda^{+}$and $\Lambda^{-}$are continuous on $(0, \infty)$. Then the distribution function $G$ of $W$ is continuous on $[a, \infty)$ for any $a>1$. Moreover, if $\bar{\Lambda}^{+}$and $\bar{\Lambda}^{-}$are such that

$-\bar{\Lambda}^{+}$and $-\bar{\Lambda}^{-}$have strictly decreasing positive derivatives $\varphi^{+}$and $\varphi^{-}$on $(0, \infty),(3.10)$ then $G$ is Lipschitz and hence absolutely continuous on $[a, \infty)$ for any $a>1$.

Proof of Lemma 3.3: Assume (3.7)-(3.9) for $r_{k}$ and $t_{k}$ as specified. Choose $1<c<d$ and apply identity (1.26) to get

$$
P\left\{\left|\frac{X\left(T\left(r_{k}\right)\right)}{r_{k}}\right| \in(c, d]\right\}=\int_{|y| \leq 1}\left(\bar{\Pi}^{+}\left(r_{k}(c-y)\right)-\bar{\Pi}^{+}\left(r_{k}(d-y)\right)\right) U_{r_{k}}\left(r_{k} \mathrm{~d} y\right)
$$


Compactness and continuity properties for two-sided Lévy exit times

$$
\begin{aligned}
& +\int_{|y| \leq 1}\left(\bar{\Pi}^{-}\left(r_{k}(c+y)\right)-\bar{\Pi}^{-}\left(r_{k}(d+y)\right)\right) U_{r_{k}}\left(r_{k} \mathrm{~d} y\right) \\
= & \int_{|y| \leq 1}\left(\Delta\left(r_{k} c, r_{k} y\right)-\Delta\left(r_{k} d, r_{k} y\right)\right) U_{r_{k}}\left(r_{k} \mathrm{~d} y\right)
\end{aligned}
$$

where for $u>0$ and $|v|<u$

$$
\Delta(u, v):=\bar{\Pi}^{+}(u-v)+\bar{\Pi}^{-}(u+v) .
$$

From (3.11) we get, using (1.25) and (1.22),

$$
\begin{gathered}
P\left\{\left|\frac{X\left(T\left(r_{k}\right)\right)}{r_{k}}\right| \in(c, d]\right\} \leq \sup _{|y| \leq 1}\left(\Delta\left(r_{k} c, r_{k} y\right)-\Delta\left(r_{k} d, r_{k} y\right)\right) E T\left(r_{k}\right) \\
\leq \frac{C_{2}}{h\left(r_{k}\right)} \sup _{|y| \leq 1}\left(\Delta\left(r_{k} c, r_{k} y\right)-\Delta\left(r_{k} d, r_{k} y\right)\right) .
\end{gathered}
$$

Applying the elementary Fact B given in the Appendix, we see that the convergence in (3.8) is uniform on $[a-1, \infty)$ for any $a>1$. Arguing via the Portmanteau theorem, as for (2.7), and with $G$ denoting the distribution function of $W$, we get from (3.7), (3.9) and (3.12),

$$
\begin{aligned}
G(d-)- & G(c)=P\{c<W<d\} \leq \limsup _{k \rightarrow \infty}\left(\frac{C_{2}}{t_{k} h\left(r_{k}\right)}\right) t_{k} \sup _{|y| \leq 1}\left(\Delta\left(r_{k} c, r_{k} y\right)-\Delta\left(r_{k} d, r_{k} y\right)\right) \\
& \leq \kappa \sup _{|y| \leq 1}\left[\left(\bar{\Lambda}^{+}(c-y)-\bar{\Lambda}^{+}(d-y)\right)+\left(\bar{\Lambda}^{-}(c+y)-\bar{\Lambda}^{-}(d+y)\right)\right],
\end{aligned}
$$

for all $0<c<d$. By assumption $\bar{\Lambda}^{ \pm}(x)$ are continuous at each $x>0$, so from inequality (3.13) it follows that $G$ is continuous on $[a, \infty)$ for any $a>1$.

Next assume, in addition, that (3.10) holds. Then, for $|y| \leq 1$ and $1<a \leq c<d$,

$$
\begin{gathered}
\bar{\Lambda}^{+}(c-y)-\bar{\Lambda}^{+}(d-y)=\int_{c-y}^{d-y} \varphi^{+}(x) \mathrm{d} x \leq(d-c) \varphi^{+}(c-y) \\
\leq(d-c) \varphi^{+}(c-1) \leq(d-c) \varphi^{+}(a-1) .
\end{gathered}
$$

Similarly for $\bar{\Lambda}^{-}$, so by inequality (3.13) and the continuity of $G$,

$$
G(d)-G(c) \leq \kappa\left(\varphi^{+}(a-1)+\varphi^{-}(a-1)\right)(d-c)=: D(a)(d-c),
$$

for all $1<a \leq c<d$. Thus $G$ is absolutely continuous on $[a, \infty)$ for all $a>1$.

Corollary 3.4. Assume $\bar{\Pi}(0+)=\infty$ and $|X(T(r))| / r \in S C$ at 0 . Then we can find sequences $r_{k} \downarrow 0$ and $t_{k} \downarrow 0$ such that (3.7)-(3.9) hold. Assume that the $\bar{\Lambda}^{+}$and $\bar{\Lambda}^{-}$in (3.8) are continuous on $(0, \infty)$. Then the $r v W$ in (3.7) has a continuous distribution $G$. Furthermore, if in addition (3.10) holds, then $G$ is Lipschitz on $[a, \infty)$ for any $a>1$.

Proof of Corollary 3.4: Assume as usual that $\bar{\Pi}(0+)=\infty$ and suppose $|X(T(r))| / r \in S C$ at 0 . Thus for every sequence $\left\{s_{j}\right\}_{j \geq 1}$ of positive numbers converging to zero there is a subsequence $\left\{r_{k}\right\}_{k \geq 1}$ such that

$$
\frac{\left|X\left(T\left(r_{k}\right)\right)\right|}{r_{k}} \stackrel{\mathrm{D}}{\longrightarrow} W
$$


where $W$ is a finite nondegenerate random variable. So (3.7) holds. By Theorem 3.1, $|X(T(r))| / r \in S C$ at 0 implies $X(t) \in F C_{0} \backslash D_{P}(N)$ at 0 . Setting $t_{k}=t\left(r_{k}\right)$, where $t(r)$ is as defined in (1.37), we also have

$$
\frac{X\left(t_{k}\right)}{r_{k}} \stackrel{\mathrm{D}}{\longrightarrow} Y
$$

for an infinitely divisible random variable $Y$. Since $X(t) \notin D_{P}(N)$ at $0, Y$ has a nontrivial Lévy measure $\Lambda$ with positive and negative tails $\bar{\Lambda}^{+}(x)$ and $\bar{\Lambda}^{-}(x), x>0$, defined in the usual way, and (3.14) implies by (1.8) that

$$
t_{k} \bar{\Pi}^{+}\left(r_{k} u\right) \rightarrow \bar{\Lambda}^{+}(u) \quad \text { and } \quad t_{k} \bar{\Pi}^{-}\left(r_{k} u\right) \rightarrow \bar{\Lambda}^{-}(u),
$$

at continuity points $u>0$ of $\Lambda^{ \pm}$. Thus (3.8) holds. Further, for this choice of $r_{k}$ and $t_{k}$, we have

$$
0<\limsup _{k \rightarrow \infty} \frac{1}{t_{k} h\left(r_{k}\right)}=\limsup _{k \rightarrow \infty} \frac{U\left(r_{k}\right)}{r_{k}^{2}} \times \frac{r_{k}^{2}}{r_{k}\left|\nu\left(r_{k}\right)\right|+U\left(r_{k}\right)} \leq 1,
$$

which proves (3.9). Under the further assumption that $\bar{\Lambda}^{ \pm}$are continuous on $(0, \infty)$, Lemma 3.3 applies to yield the continuity of $G$, and if in addition $\Lambda^{ \pm}$satisfy (3.10), $G$ has the claimed Lipschitz property, again by Lemma 3.3.

Following Lemma 3.3, we are led to ask what added conditions are needed to ensure that for each $a>1, \bar{\Lambda}^{+}$and $\bar{\Lambda}^{-}$have strictly decreasing positive derivatives on $(0, \infty)$. It turns out that self-decomposable Lévy processes have this property. Recall that a random variable $Y$ is said to be self-decomposable if its c.f. is of the form

$$
E e^{\mathrm{i} \theta Y}=\exp \left(\mathrm{i} \gamma_{Y} \theta-\frac{1}{2} A_{Y} \theta^{2}+\int_{\mathbb{R} \backslash\{0\}}\left(e^{\mathrm{i} x \theta}-1-\mathrm{i} \theta x \mathbf{1}\{|x| \leq 1\}\right) \frac{k(x)}{|x|} \mathrm{d} x\right),
$$

where $\gamma_{Y} \in \mathbb{R}, A_{Y} \geq 0, k(x) \geq 0, \int_{\mathbb{R} \backslash\{0\}}\left(|x|^{2} \wedge 1\right) k(x) \mathrm{d} x /|x|<\infty$, and $k(x)$ is increasing on $(-\infty, 0)$ and decreasing on $(0, \infty)$. See Corollary 15.11, p.95, of [21]. Such a $Y$ is clearly inf. div. and the Lévy process $(Y(t))_{t \geq 0}$ such that $Y(1) \stackrel{\mathrm{D}}{=} Y$ is said to be a self-decomposable Lévy process.

We need the following result.

Proposition 3.5. Let $(X(t))_{t \geq 0}$ be a self-decomposable Lévy process and $b(t), t>0$, a positive norming function such that for a sequence $t_{k} \downarrow 0, X\left(t_{k}\right) / b\left(t_{k}\right)$ converges in distribution to a nondegenerate inf. div. random variable $Y$. Let $(Y(t))_{t \geq 0}$ be the Lévy process with $Y(1) \stackrel{\mathrm{D}}{=} Y$. Then $(Y(t))_{t \geq 0}$ is a self-decomposable Lévy process.

Proof of Proposition 3.5: Since $X(t)$ is a self-decomposable Lévy process, $X(1)$ has a c.f. of the form (3.15), with $\gamma_{Y}$ replaced by $\gamma_{X} \in \mathbb{R}$ and $A_{Y}$ replaced by $A_{X} \geq 0$. Then $X\left(t_{k}\right) / b\left(t_{k}\right)$ is a self-decomposable inf. div. random variable with triplet $\left(\gamma_{k}, \sigma_{k}^{2}, \Pi_{k}\right)$, where for each $k \geq 1$

$$
\sigma_{k}^{2}=\frac{t_{k} A_{X}}{b_{k}^{2}}, \gamma_{k}=\frac{\gamma_{X} t_{k}}{b_{k}}+\int_{\mathbb{R} \backslash\{0\}} x\left(\mathbf{1}_{\{|x| \leq 1\}}-\mathbf{1}_{\left\{\left|b_{k} x\right| \leq 1\right\}}\right) \Pi_{k}(\mathrm{~d} x),
$$

and $\Pi_{k}(\mathrm{~d} x)$ is the Lévy measure $t_{k} \Pi\left(b_{k} \mathrm{~d} x\right)$, with $\Pi(\mathrm{d} x)=k(x) \mathrm{d} x /|x|, x \in \mathbb{R}$.

We record here the fact that $\Pi$ is the Lévy measure of a self-decomposable distribution if and only if the functions

$$
\bar{\Pi}^{+}\left(e^{-s}\right) \text { and } \bar{\Pi}^{-}\left(e^{-s}\right), s \in \mathbb{R},
$$


are convex on $\mathbb{R}$. For this see Theorem 4.1 in Barndorff-Nielsen and Shephard (2001) and the references therein.

Now for any $z>0$ and $\rho>0$

$$
\bar{\Pi}^{+}(\rho z)=\int_{(\rho z, \infty)} \frac{k(x)}{x} \mathrm{~d} x=\int_{(z, \infty)} \frac{k(\rho x)}{x} \mathrm{~d} x=: \bar{\Pi}_{\rho}^{+}(z)
$$

and

$$
\bar{\Pi}^{-}(\rho z)=\int_{(\rho z, \infty)} \frac{k(-x)}{x} \mathrm{~d} x=\int_{(z, \infty)} \frac{k(-\rho x)}{x} \mathrm{~d} x=: \bar{\Pi}_{\rho}^{-}(z) .
$$

Clearly for any $\rho>0$ both

$$
h_{\rho}^{+}(s)=\bar{\Pi}_{\rho}^{+}\left(e^{-s}\right) \text { and } h_{\rho}^{-}(s)=\bar{\Pi}_{\rho}^{-}\left(e^{-s}\right)
$$

are convex on $\mathbb{R}$. Using Fact $C$ in the Appendix we can show that whenever there exists a sequence of positive constants $\left\{\rho_{k}\right\}_{k>1}$ converging to zero and a sequence of positive constants $\left\{a_{k}\right\}_{k>1}$ such that both $a_{k} h_{\rho_{k}}^{+}(s)$ and $a_{k} h_{\rho_{k}}^{-}(s)$ converge to a finite limit on a dense subset of $\mathbb{R}$, they each converge to a convex function uniformly on compact subsets of $\mathbb{R}$.

Now assume that

$$
\frac{X\left(t_{k}\right)}{b\left(t_{k}\right)} \stackrel{\mathrm{D}}{\longrightarrow} Y(1),
$$

where $Y(1)$ is a nondegenerate inf. div. random variable with Lévy measure $\Lambda$. Then by (1.8) of Subsection 1.3, for every continuity point $u$ of $\bar{\Lambda}^{ \pm}$,

$$
t_{k} \bar{\Pi}^{ \pm}\left(b\left(t_{k}\right) u\right) \rightarrow \bar{\Lambda}^{ \pm}(u) .
$$

Setting $a_{k} h_{\rho_{k}}^{ \pm}(s)=t_{k} \bar{\Pi}^{ \pm}\left(b\left(t_{k}\right) e^{-s}\right)$ for $s \in \mathbb{R}$, we see that (3.19) in combination with the above mentioned Fact $\mathrm{C}$ finishes the proof, since both $\bar{\Lambda}^{+}\left(e^{-s}\right)$ and $\bar{\Lambda}^{-}\left(e^{-s}\right)$ are convex on $\mathbb{R}$, which implies that $Y(1)$ is a self-decomposable inf. div. random variable.

Theorem 3.6. Suppose $X$ is a self-decomposable Lévy process such that $|X(T(r))| / r$ is in $S C$ at 0 . Then every subsequential limit $r v W$ of $\left|X\left(T\left(r_{k}\right)\right)\right| / r_{k}$ along sequences $r_{k} \downarrow 0$, as $k \rightarrow \infty$, has a distribution function $G$ that is absolutely continuous on $[a, \infty)$ for any $a>1$; further, $G(1)-G(1-)=G(1)<1$.

Proof of Theorem 3.6: Assume that $X$ is a self-decomposable Lévy process such that $|X(T(r))| / r$ is in $S C$ at 0 . Then by Theorem $3.1 X \in F C_{0}$ with a norming function $b(t)$ which we can assume satisfies (1.17), and $X(t) \notin D_{P}(N)$ at 0 . So necessarily $A_{X}=0$ in its c.f. By Proposition 3.5, each subsequential limit law $G$ of $X(t) / b(t)$ is self-decomposable with a necessarily nontrivial Lévy measure $\Lambda$ of the form (3.15) with $A_{Y}=0$. To see why $A_{X}=A_{Y}=0$ refer to Fact D in the Appendix. It follows that

$$
-\frac{\mathrm{d}}{\mathrm{d} x} \bar{\Lambda}^{+}(x)=-\frac{\mathrm{d}}{\mathrm{d} x} \int_{x}^{\infty} \frac{k(y)}{y} \mathrm{~d} y=\frac{k(x)}{x}
$$

is strictly decreasing on $(0, \infty)$. Similarly $-\mathrm{d} \bar{\Lambda}^{-}(x) / \mathrm{d} x$ is strictly decreasing on $(0, \infty)$. Consequently $G$ is absolutely continuous on $[a, \infty)$ for any $a>1$ by Lemma 3.5.

It remains to note that $G(1)-G(1-)=G(1)<1$ because clearly $G$ attributes positive mass to $(1, \infty)$, completing the proof of Theorem 3.6. 
Example 3.7. Suppose $(X(t))_{t>0}$ is a subordinator in the domain of attraction of a stable law of index $0<\alpha<1$ as $t \downarrow 0$, hence, in $F C_{0} \backslash D_{P}(N)$. Then

$$
\frac{X(T(r))}{r} \stackrel{\mathrm{D}}{\rightarrow} W, \text { as } r \downarrow 0,
$$

where for $w>1$

$$
G(w)=P\{W \leq w\}=\frac{\sin \alpha \pi}{\pi} \int_{1}^{w}(x-1)^{-\alpha} x^{-1} \mathrm{~d} x
$$

When $X$ is in fact the stable subordinator of index $\alpha$, this follows by setting $\rho=1, a=r$ and $b=r w$, where $w>1$, in Ex.3, p.238 of [2] and changing the variable, or similarly from Ex. 44.24, p. 331, of [21]. Clearly $G$ is Lipschitz on $[a, \infty)$ for any $a>1$.

An analogous result holds for $X(T(r)-)$, the position of $X$ before exit. In this case all subsequential limit laws of $|X(T(r)-)| / r$ have support contained in [0,1]. Again supposing $X$ is a stable subordinator of index $0<\alpha<1$, we have as $r \downarrow 0$

$$
\frac{X(T(r)-)}{r} \stackrel{\mathrm{D}}{\rightarrow} W, \text { as } r \downarrow 0,
$$

where $W$ is the generalized standard arcsine random variable with density $f(w ; \alpha)$ defined by

$$
\frac{\sin \alpha \pi}{\pi} w^{\alpha-1}(1-w)^{-\alpha}, \text { for } 0<w<1 .
$$

For this result see Thm. 6, p.81, of [2]. This example agrees with Theorem 2.4.

\section{The jump causing ruin}

In this section we confine our attention to $\Delta X(T(r))$. We say that $|\Delta X(T(r))| / r$ is stochastically compact at 0 (is in $S C$ at 0 ) if every sequence $r_{k} \downarrow 0$ has a subsequence $r_{k^{\prime}}$ such that $\left|\Delta X\left(T\left(r_{k^{\prime}}\right)\right)\right| / r_{k^{\prime}}$ converges in distribution to a finite nondegenerate random variable.

Theorem 4.1. Assume $\bar{\Pi}(0+)=\infty$.

(i) $|\Delta X(T(r))| / r$ is tight as $r \downarrow 0$ if and only if $|X(T(r))| / r$ is tight as $r \downarrow 0$.

(ii) $|\Delta X(T(r))| / r$ is in $S C$ at 0 if and only if $|X(T(r))| / r$ is in $S C$ at 0 .

Proof of Theorem 4.1: (i) Since $|X(T(r)-)| \leq r \leq|X(T(r))|$ and

$$
\frac{|X(T(r))|}{r}-1 \leq \frac{|\Delta X(T(r))|}{r}=\left|\frac{X(T(r))}{r}-\frac{X(T(r)-)}{r}\right| \leq \frac{|X(T(r))|}{r}+1,
$$

we see that $|\Delta X(T(r))| / r$ is tight as $r \downarrow 0$ iff $|X(T(r))| / r$ is tight as $r \downarrow 0$.

(ii) Suppose $|\Delta X(T(r))| / r$ is in $S C$ at 0 . Then by Part (i) $|X(T(r))| / r$ is tight at 0 , so (3.1) holds. Suppose (1.49) holds. By Theorem 1.8 this happens if and only there is a sequence $r_{k} \downarrow 0$ such that for all $c>0$

$$
P\left(\frac{\left|\Delta X\left(T\left(r_{k}\right)\right)\right|}{r_{k}}>c\right) \rightarrow 0, \text { as } k \rightarrow \infty
$$

which implies that

$$
\frac{\left|\Delta X\left(T\left(r_{k}\right)\right)\right|}{r_{k}} \rightarrow 0, \text { as } k \rightarrow \infty
$$


This contradicts the assumption that $|\Delta X(T(r))| / r$ is in $S C$ at 0 . So (1.49) cannot hold and hence we must have (2.1). Thus by Theorem 3.1, $|X(T(r))| / r$ is in $S C$ at 0 .

Conversely if $|X(T(r))| / r$ is in $S C$ at 0 then $|X(T(r))| / r$ is tight so $|\Delta X(T(r))| / r$ is tight. We claim there can be no sequence $r_{k} \downarrow 0$ and constant $b \geq 0$ such that

$$
\frac{\left|\Delta X\left(T\left(r_{k}\right)\right)\right|}{r_{k}} \rightarrow b, \text { as } k \rightarrow \infty,
$$

since this would imply that (4.1) is satisfied for some $c>b$, which by Theorem 1.8 implies (1.49). But again this contradicts (2.1), so by Theorem 3.1, (4.2) cannot happen. Thus $|\Delta X(T(r))| / r$ is in $S C$ at 0.

Lemma 4.2. Assume $\bar{\Pi}(0+)=\infty$. Suppose for a sequence $r_{k} \downarrow 0$

$$
\frac{\left|\Delta X\left(T\left(r_{k}\right)\right)\right|}{r_{k}} \stackrel{\mathrm{D}}{\longrightarrow} D,
$$

where $D$ is a nondegenerate random variable. Suppose there exist nonnegative continuous decreasing functions $\bar{\Lambda}^{+}$and $\bar{\Lambda}^{-}$on $(0, \infty)$, which vanish at infinity, and a sequence of positive constants $\left\{t_{k}\right\}_{k \geq 1}$, such that for all $u>0$, (3.8) and (3.9) hold. Then the distribution function $H$ of $D$ is continuous on $(0, \infty)$. Moreover, if $\bar{\Lambda}^{+}$and $\bar{\Lambda}^{-}$are such that (3.10) is satisfied, then $H$ is Lipschitz and hence absolutely continuous on $[a, \infty)$ for any $a>0$.

Proof of Lemma 4.2: We get, using (1.33), (1.25) and (1.22), that

$$
\begin{aligned}
P\left\{\left|\frac{\Delta X\left(T\left(r_{k}\right)\right)}{r_{k}}\right|\right. & \in(c, d)\} \leq t_{k}\left(\bar{\Pi}\left(r_{k} c\right)-\bar{\Pi}\left(r_{k} d\right)\right) t_{k}^{-1} U_{r_{k}}\left(\left[-r_{k}, r_{k}\right]\right) \\
& \leq t_{k}\left(\bar{\Pi}\left(r_{k} c\right)-\bar{\Pi}\left(r_{k} d\right)\right) E T\left(r_{k}\right) \\
& \leq t_{k}\left(\bar{\Pi}\left(r_{k} c\right)-\bar{\Pi}\left(r_{k} d\right)\right) \frac{t_{k}^{-1} C_{2}}{h\left(r_{k}\right)}
\end{aligned}
$$

Now the remainder of the proof is very similar to that of Lemma 3.3, so the details are omitted.

Remark 4.3. Assume $\bar{\Pi}(0+)=\infty$ and $X(t) \in F C_{0} \backslash D_{P}(N)$ at 0 , so that both $|X(T(r))| / r$ and $|\Delta X(T(r))| / r$ are in $S C$ at 0 . Let $t(r)=r^{2} / U(r)$, for $r>0$. By arguing as in Corollary 3.4 , for every sequence $s_{j}, j \geq 1$, of positive numbers converging to zero there is a subsequence $r_{k}, k \geq 1$, such that for nondegenerate random variables $D$ and $W$

$$
\frac{\left|\Delta X\left(T\left(r_{k}\right)\right)\right|}{r_{k}} \stackrel{\mathrm{D}}{\longrightarrow} D, \quad \text { and } \quad \frac{\left|X\left(T\left(r_{k}\right)\right)\right|}{r_{k}} \stackrel{\mathrm{D}}{\longrightarrow} W,
$$

and, with $t_{k}=t\left(r_{k}\right)$,

$$
\frac{X\left(t_{k}\right)}{r_{k}} \stackrel{\mathrm{D}}{\longrightarrow} Y
$$

for an infinitely divisible random variable $Y$ with a nontrivial Lévy measure $\Lambda$. Then if $\bar{\Lambda}^{+}$ and $\bar{\Lambda}^{-}$are continuous, (4.4) implies that (3.8) holds. Also it was pointed out in Corollary 3.4 that $X(t) \in F C_{0} \backslash D_{P}(N)$ at 0 implies that (3.9) is satisfied. Hence, if $\bar{\Lambda}^{+}$and $\bar{\Lambda}^{-}$are continuous on $(0, \infty)$ or satisfy (3.10), then Lemma 4.2 applies. 


\section{The symmetric St. Petersburg process}

This interesting process is an example of an $X(t)$ in $F C_{0}$ such that the Lévy measure of any of its subsequential limit rvs is not continuous. Consider the St. Petersburg game-type Lévy tail functions:

$$
\bar{R}^{+}(x)=R\{(x, \infty)\}=\bar{R}^{-}(x)=R\{(-\infty,-x)\}=2^{-\left\lfloor\log _{2}(x)\right\rfloor}, x>0,
$$

and set

$$
\bar{R}(x)=\bar{R}^{+}(x)+\bar{R}^{-}(x)=2 \bar{R}^{+}(x) .
$$

Let $R$ be the corresponding measure and notice that $\bar{R}(0+)=\infty$. Let $X=(X(t))_{t \geq 0}$ be the symmetric St. Petersburg Lévy process with Lévy triplet $(0,0, R(\mathrm{~d} x))$. Then, defining a centering function as in (1.3), by symmetry we have, for all $x>0$,

$$
\nu_{R}(x)=0 .
$$

Since

$$
\frac{x}{2} \leq 2^{\left\lfloor\log _{2}(x)\right\rfloor} \leq x \quad \text { and so } \quad \frac{1}{x} \leq \frac{1}{2} \bar{R}(x)=2^{-\left\lfloor\log _{2}(x)\right\rfloor} \leq \frac{2}{x},
$$

we have for $x>0$

$$
2 \int_{0}^{x} y \bar{R}(y) \mathrm{d} y \geq 4 \int_{0}^{x} \mathrm{~d} y=4 x \geq x^{2} \bar{R}(x),
$$

hence by (1.14), $X(t) \in F C_{0}$ at 0 .

Define the norming function

$$
b(t)=2^{\left\lfloor\log _{2}(t)\right\rfloor}, t>0 .
$$

Then for integers $k \geq 1$ and $t>0$ such that $2^{-k} \leq t<2^{-k+1}$ we have $b(t)=2^{-k}$. Further, for integers $m \geq 1$ and $u>0$ such that $2^{-m} \leq u<2^{-m+1}$, we have

$$
\bar{R}^{ \pm}(b(t) u)=\bar{R}^{ \pm}\left(b\left(2^{-k}\right) u\right)=2^{m+k}
$$

Now fix $\lambda \in[1,2]$ and take $t_{k}=t_{k}(\lambda)$ such that $2^{-k} \leq t_{k}<2^{-k+1}$ and $t_{k} 2^{k} \rightarrow \lambda$. Then for all $u>0$ such that $2^{-m} \leq u<2^{-m+1}$ we have

$$
2^{m+k}=2^{m} b\left(t_{k}\right) \leq u b\left(t_{k}\right)<2^{m+1} b\left(t_{k}\right)=2^{m+k+1}
$$

and consequently

$$
t_{k} \bar{R}^{ \pm}\left(b\left(t_{k}\right) u\right) \rightarrow \lambda 2^{m}=\lambda \bar{R}^{ \pm}(u) .
$$

Next take $u \in(0,1]$ and consider

$$
\frac{t_{k}}{b^{2}\left(t_{k}\right)} \int_{-u b\left(t_{k}\right)}^{u b\left(t_{k}\right)} x^{2} R(\mathrm{~d} x)=-u^{2} t_{k} \bar{R}\left(b\left(t_{k}\right) u\right)+2 t_{k} \int_{0}^{1} x \bar{R}\left(b\left(t_{k}\right) x\right) \mathrm{d} x .
$$

The first term on the RHS tends to $-\lambda u^{2} \bar{R}(u)$ as $k \rightarrow \infty$, by (5.4). In the second term on the RHS, by (5.2), we have

$$
t_{k} x \bar{R}\left(b\left(t_{k}\right) x\right) \leq \frac{4 t_{k} x}{x b\left(t_{k}\right)} \leq 2 t_{k} x \bar{R}\left(b\left(t_{k}\right)\right) \leq 2 \lambda,
$$

for $k$ large enough, not depending on $x$. So we can apply dominated convergence in the integral on the RHS of (5.5), and deduce that, for $0<u \leq 1$,

$$
\lim _{k \rightarrow \infty} \frac{t_{k}}{b^{2}\left(t_{k}\right)} \int_{-u b\left(t_{k}\right)}^{u b\left(t_{k}\right)} x^{2} R(\mathrm{~d} x)=-\lambda u^{2} \bar{R}(u)+2 \lambda \int_{0}^{u} x \bar{R}(x) \mathrm{d} x=\lambda \int_{-u}^{u} x^{2} R(\mathrm{~d} x) .
$$


When $u>1$ we write

$$
\frac{t_{k}}{b^{2}\left(t_{k}\right)} \int_{-u b\left(t_{k}\right)}^{u b\left(t_{k}\right)} x^{2} R(\mathrm{~d} x)=\frac{t_{k}}{b^{2}\left(t_{k}\right)}\left(\int_{-b\left(t_{k}\right)}^{b\left(t_{k}\right)}+\int_{1<|y|<u b\left(t_{k}\right)}\right) x^{2} R(\mathrm{~d} x) .
$$

On the RHS the first integral tends to $\lambda \int_{-1}^{1} x^{2} R(\mathrm{~d} x)$ by (5.6). Apply (5.4) and dominated convergence to the second integral to get the limit $\lambda \int_{1<|y|<u} x^{2} R(\mathrm{~d} x)$. Adding this to the first expression we see that (5.6) in fact holds for all $u>0$.

Taking together (5.4), (5.6) and (5.1), we see from the convergence criteria in Subsection 1.3 that $X\left(t_{k}\right) / b\left(t_{k}\right) \stackrel{\mathrm{D}}{\longrightarrow} Y_{\lambda}$ as $k \rightarrow \infty$, where $Y_{\lambda}$ has Lévy triplet $(0,0, \lambda R(\mathrm{~d} x))$.

Now set $\mathcal{Y}=\left\{Y_{\lambda}: \lambda \in[1,2]\right\}$. We pointed out above that the symmetric St. Petersburg Lévy process $(X(t))_{t>0}$ is in the centered Feller class at 0 (at $\left.\infty\right)$. It is readily shown that every random variable in $\mathcal{Y}$ is obtainable as a distributional limit of $X\left(t_{k}\right) / b\left(t_{k}\right)$ for an appropriately chosen sequence of positive constants $\left\{t_{k}\right\}$. But the Lévy tail function $\bar{R}(x)$ is obviously not continuous on $[a-1, \infty)$ for any $a>1$.

\section{Appendix: Proofs of facts A, B, C and D}

\subsection{Fact A}

We prove here Fact A: A nondegenerate Lévy process $X(t)$ with infinite Lévy measure $\Pi$ is in $F C$ at 0 whenever (3.4) holds.

Proof of Fact A: We can assume $\sigma^{2}=0$, since whenever $\sigma^{2}>0$, there exists a positive norming function $b(t)$ such that

$$
\frac{X(t)}{b(t)} \stackrel{\mathrm{D}}{\longrightarrow} Z \text {, as } t \downarrow 0,
$$

where $Z$ is a standard normal random variable. (See [15].) In this case there is nothing to prove, since $X(t)$ is already in $F C$ at 0 and condition (3.4) is irrelevant.

The proof that follows is a modification of arguments in [6]. For $\lambda>0$ let

$$
\limsup _{t \downarrow 0} \frac{\bar{\Pi}(t \lambda)}{\bar{\Pi}(t)}=\varphi(\lambda)
$$

Without loss of generality we can assume that $\varphi(\lambda)$ is finite and positive for all $\lambda>0$. By a standard argument, (3.4) implies

$$
\lim _{\lambda \rightarrow \infty} \limsup _{x \downarrow 0} \frac{\bar{\Pi}(\lambda x)}{\bar{\Pi}(x)}=0,
$$

hence $\varphi(\lambda) \rightarrow 0$ as $\lambda \rightarrow \infty$. From (6.1) we get for all $0<x<1$

$$
\liminf _{t \downarrow 0} \frac{\bar{\Pi}(t)}{\bar{\Pi}(t / x)}=\liminf _{t \downarrow 0} \frac{\bar{\Pi}(t x)}{\bar{\Pi}(t)}=\frac{1}{\varphi(1 / x)}=: \psi(x) \geq 1,
$$

which gives by Fatou's lemma

$$
\liminf _{t \downarrow 0} \frac{\int_{0}^{t} y \bar{\Pi}(y) \mathrm{d} y}{t^{2} \bar{\Pi}(t)}=\liminf _{t \downarrow 0} \frac{\int_{0}^{1} x \bar{\Pi}(t x) \mathrm{d} x}{\bar{\Pi}(t)} \geq \int_{0}^{1} x \psi(x) \mathrm{d} x .
$$

Clearly $\psi(x)=1 / \varphi(1 / x) \rightarrow \infty$ as $x \downarrow 0$. Thus for any $M>1$ there exists $x_{0} \in(0,1)$ such that $\psi(x)>M$ for all $0<x<x_{0}$. This implies that

$$
\int_{0}^{1} x \psi(x) \mathrm{d} x \geq \int_{0}^{x_{0}} x M \mathrm{~d} x+\int_{x_{0}}^{1} x \mathrm{~d} x=\frac{1}{2} x_{0}^{2}(M-1)+\frac{1}{2}>\frac{1}{2} .
$$


Thus, keeping in mind that $\sigma^{2}=0$,

$$
\liminf _{t \downarrow 0}\left(\frac{1}{2}+\frac{V(t)}{2 t^{2} \bar{\Pi}(t)}\right)=\liminf _{t \downarrow 0} \frac{\int_{0}^{t} y \bar{\Pi}(y) \mathrm{d} y}{t^{2} \bar{\Pi}(t)}>\frac{1}{2},
$$

and thus (1.14) holds, i.e.

$$
\liminf _{t \downarrow 0} \frac{V(t)}{t^{2} \bar{\Pi}(t)}>0 .
$$

This implies that $X(t) \in F C$ at 0 .

\subsection{Fact B}

We need the following elementary Fact B. Its proof is very similar to that of Polya's theorem (see Theorem B.7.7 of [3]).

Let $\left(f_{k}\right)_{k>1}$ be a sequence of decreasing functions on $(0, \infty)$ such that for each $x \in(0, \infty), f_{k}(x) \rightarrow f(x)$, where $f$ is a continuous function on $(0, \infty)$ that vanishes at infinity. Then for all $c>0, \sup _{x \geq c}\left|f_{k}(x)-f(x)\right| \rightarrow 0$.

Proof of Fact B:. Choose any $\varepsilon>0$ and $C>0$ such that $|f(x)| \leq \varepsilon / 4$ for all $x \geq C$. Select $c \in(0, C)$. Since $f$ is uniformly continuous on $[c, C]$, we can select $m \geq 1$ and $c=c_{0}<c_{1}<\cdots<c_{m}<c_{m+1}=C$ such that

$$
\max _{0 \leq n \leq m} \sup \left\{|f(x)-f(y)|: x, y \in\left[c_{n}, c_{n+1}\right]\right\} \leq \varepsilon / 8 .
$$

Now for any $k \geq 1,0 \leq n \leq m$ and $z \in\left[c_{n}, c_{n+1}\right]$,

$$
f_{k}\left(c_{n+1}\right) \leq f_{k}(z) \leq f_{k}\left(c_{n}\right) .
$$

Thus for all $x, y \in\left[c_{n}, c_{n+1}\right]$

$$
\left|f_{k}(y)-f_{k}(x)\right| \leq\left|f_{k}\left(c_{n}\right)-f_{k}\left(c_{n+1}\right)\right| .
$$

Choose $K \geq 1$ so large such that for all $k \geq K$

$$
\max _{0 \leq n \leq m}\left|f_{k}\left(c_{n}\right)-f\left(c_{n}\right)\right| \leq \varepsilon / 8 .
$$

We get then that for all $k \geq K, 0 \leq n \leq m$ and $x \in\left[c_{n}, c_{n+1}\right]$,

$$
\begin{gathered}
\left|f(x)-f_{k}(x)\right| \leq\left|f\left(c_{n}\right)-f_{k}(x)\right|+\left|f\left(c_{n}\right)-f(x)\right| \\
\leq\left|f\left(c_{n}\right)-f_{k}\left(c_{n}\right)\right|+\left|f\left(c_{n}\right)-f\left(c_{n+1}\right)\right| \leq \varepsilon / 4 .
\end{gathered}
$$

This gives for all $k \geq K$

$$
\begin{gathered}
\sup _{x \geq c}\left|f_{k}(x)-f(x)\right| \leq \max _{0 \leq n \leq m} \sup _{x \in\left[c_{n}, c_{n+1}\right]}\left|f_{k}(x)-f(x)\right|+|f(C)|+\left|f_{k}(c)\right| \\
\leq 2 \max _{0 \leq n \leq m} \sup _{x \in\left[c_{n}, c_{n+1}\right]}\left|f_{k}(x)-f(x)\right|+2|f(C)| \leq \varepsilon
\end{gathered}
$$

as required.

\subsection{Fact C}

The following Fact $\mathrm{C}$ is a consequence of Exercise 7, p.20, of [20]: let $\left(f_{k}\right)_{k>1}$ be a sequence of convex functions on $\mathbb{R}$ that converges to a finite limit on a dense subset of $\mathbb{R}$. Then $f_{k}(x)$ converges for all $x \in \mathbb{R}$ to a convex function and the convergence is uniform on compact subsets of $\mathbb{R}$. 


\subsection{Fact D}

The following Fact D is used in the proof of Theorem 3.6: Suppose $X(t)$ is a Lévy process with triplet $\left(\gamma, \sigma^{2}, \Pi\right)$ and for $t_{k} \downarrow 0, a_{k} \in \mathbb{R}$ and $b_{k}>0$, (1.7) holds where $Y$ is an inf. div. $r v$ with triplet $\left(\beta, \tau^{2}, \Lambda\right)$ such that $\beta \in \mathbb{R}, \tau^{2} \geq 0$, and $\Lambda$ is a Lévy measure on $\mathbb{R}$. If $\sigma^{2}>0$ or $\tau^{2}>0$, then $X(t) \in D_{P}(N)$.

Proof of Fact D:. We keep the notation of Subsection 1.3.

Case 1. Suppose $\sigma^{2}>0$, then since $x^{2} \bar{\Pi}(x) \rightarrow 0$ as $x \downarrow 0$,

$$
\limsup _{x \downarrow 0} x^{2} \bar{\Pi}(x) / V(x) \leq \limsup _{x \downarrow 0} x^{2} \bar{\Pi}(x) / \sigma^{2}=0 .
$$

Case 2. Suppose $\tau^{2}>0$. Then by (1.8), for every continuity point $h$ of $\bar{\Lambda}$,

$$
\frac{h^{2} b_{k}^{2} \bar{\Pi}\left(h b_{k}\right)}{V\left(h b_{k}\right)}=\frac{h^{2} t_{k} \bar{\Pi}\left(h b_{k}\right)}{t_{k} V\left(h b_{k}\right) / b_{k}^{2}} \rightarrow \frac{h^{2} \bar{\Lambda}(h)}{\tau^{2}+\int_{0<|x| \leq h} x^{2} \Lambda(\mathrm{d} x)} \leq \frac{h^{2} \bar{\Lambda}(h)}{\tau^{2}} .
$$

Since $h^{2} \bar{\Lambda}(h) / \tau^{2}$ converges to 0 as $h \downarrow 0$, we can argue that

$$
\liminf _{x \downarrow 0} x^{2} \bar{\Pi}(x) / V(x)=0 .
$$

Thus in either case, by Theorem 1.2, $X(t) \in D_{P}(N)$.

Acknowledgments. We are grateful to a referee for a close and careful reading of the paper which enabled us to make some significant clarifications.

\section{References}

[1] Barndorff-Nielsen, O.E. and Shephard, N. (2001) Modelling by Lévy processes. Selected Proceedings of the Symposium on Inference for Stochastic Processes (Athens, GA, 2000), 2531, IMS Lecture Notes Monogr. Ser., 37, Inst. Math. Statist., Beachwood, OH. MR-2002501

[2] Bertoin, J. (1996) Lévy Processes. Cambridge Tracts in Mathematics, 121. Cambridge University Press, Cambridge. MR-1406564

[3] Bickel, P.J. and Doksum, K.A. (2015) Mathematical Statistics-Basic Ideas and Selected Topics. Vol. 1. 2nd Ed. Texts in Statistical Science Series. CRC Press, Boca Raton, FL, 2015. MR3445928

[4] Billingsley, P. (1999) Convergence of Probability Measures, Wiley series in probability and statistics, 2nd Ed. MR-1700749

[5] Buchmann, B., Maller, R.A. and Mason, D.M. (2015) Laws of the iterated logarithm for selfnormalised Lévy processes at zero. Trans. Amer. Math. Soc. 367, 1737-1770. MR-3286497

[6] de Haan, L. and Ridder, G. (1979) Stochastic compactness of sample extremes. Ann. Probab., 7, 290-303. MR-0525055

[7] Doney, R.A. and Maller, R.A. (2002) Stability of the overshoot for Lévy processes. Ann. Probab. 30, 188-212. MR-1894105

[8] Erickson, K. and Maller, R. (2007) Finiteness of integrals of functions of Lévy processes, Proc. London Math. Soc., 94, 386-420. MR-2308232

[9] Feller, W. (1965-66) On regular variation and local limit theorems, Proc. V Berkeley Symp. Math. Stats. Prob. II, Part I, 373-388. MR-0219117

[10] Griffin, P.S. and Maller, R.A. (1999) On compactness properties of the exit position of a random walk from an interval. Proc. London Math. Soc., 78, 459-480. MR-1665250

[11] Griffin, P.S. and McConnell, T.R. (1992) On the position of a random walk at the time of first exit from a sphere, Ann. Prob., 20, 825-854. MR-1159576

[12] Griffin, P.S. and McConnell, T.R. (1994) Gambler's ruin and the first exit position of random walk from large spheres. Ann. Prob. 22, 1429-1472. MR-1303650 
[13] Kallenberg, O. (2002) Foundations of Modern Probability, Springer, New York, Berlin, Heidelberg. MR-1876169

[14] Maller, R.A. (2009) Small-time versions of Strassen's law for Lévy processes, Proc. London Math. Soc., 98, 531-558. MR-2481958

[15] Maller, R.A. and Mason, D.M. (2010) Small-time compactness and convergence behaviour of deterministically and self-normalised Lévy processes, Trans. Amer. Math. Soc., 362, 22052248. MR-2574893

[16] Maller, R.A. and Mason, D.M. (2013) A characterization of small and large time limit laws for self-normalized Lévy processes. In: Limit theorems in probability, statistics and number theory, 141-169, Springer Proc. Math. Stat., 42, Springer, Heidelberg. MR-3079142

[17] Maller, R.A. and Mason, D.M. (2015) Matrix normalized convergence of a Lévy process to normality at zero, Stoch. Proc. Appl., 125, 2353-2382. MR-3322867

[18] Maller, R.A. and Mason, D.M. (2018) Matrix normalised stochastic compactness for a Lévy process at zero. Electron. J. Probab. 23, Paper No. 69. MR-3835475

[19] Pruitt, W.E. (1981) The growth of random walks and Lévy processes. Ann. Probab. 9, 948956.MR-0632968

[20] Roberts, A.W and Varberg, D.E. (1973) Convex functions. Pure and Applied Mathematics, Vol. 57. Academic Press. MR-0442824

[21] Sato, Ken-iti (1999) Lévy Processes and Infinitely Divisible Distributions. Cambridge Studies in Advanced Mathematics, 68. Cambridge University Press, Cambridge. MR-3185174 